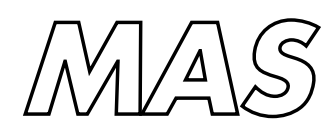

Modelling, Analysis and Simulation

Modelling, Analysis and Simulation
MAS $\begin{gathered}\text { Fourier two-level analysis for higher dimensional } \\ \text { discontinuous Galerkin discretisation }\end{gathered}$

P.W. Hemker, M.H. van Raalte

Report MAS-R0227 NOVember 30, 2002 
CWI is the National Research Institute for Mathematics and Computer Science. It is sponsored by the Netherlands Organization for Scientific Research (NWO).

$\mathrm{CWI}$ is a founding member of ERCIM, the European Research Consortium for Informatics and Mathematics.

CWI's research has a theme-oriented structure and is grouped into four clusters. Listed below are the names of the clusters and in parentheses their acronyms.

Probability, Networks and Algorithms (PNA)

Software Engineering (SEN)

Modelling, Analysis and Simulation (MAS)

Information Systems (INS)

Copyright @ 2001, Stichting Centrum voor Wiskunde en Informatica

P.O. Box 94079, 1090 GB Amsterdam (NL)

Kruislaan 413, 1098 SJ Amsterdam (NL)

Telephone +31205929333

Telefax +31205924199

ISSN 1386-3703 


\title{
Fourier Two-Level Analysis for Higher Dimensional Discontinuous Galerkin Discretisation
}

\author{
P.W. Hemker and M.H. van Raalte \\ KdV Institute for Mathematics, University of Amsterdam \\ Plantage Muidergracht 24, 1018 TV Amsterdam, The Netherlands \\ and \\ CWI \\ P.O. Box 94079, 1090 GB Amsterdam, The Netherlands
}

\begin{abstract}
In this paper we study the convergence of a multigrid method for the solution of a two-dimensional linear second order elliptic equation, discretized by discontinuous Galerkin (DG) methods. For the Baumann-Oden and for the symmetric DG method, we give a detailed analysis of the convergence for cell- and point-wise block-relaxation strategies.

We show that, for a suitably constructed two-dimensional polynomial basis, point-wise block partitioning gives much better results than the classical cell-wise partitioning. Independent of the mesh size, for Poisson's equation, simple MG cycles, with block Gauss Seidel and symmetric block Gauss Seidel smoothing, yield a convergence rate of $0.4-0.6$ per iteration sweep for both DG-methods studied.
\end{abstract}

2000 Mathematics Subject Classification: 65F10, 65N12, 65N15, 65N30, 65N55

Keywords and Phrases: Discontinuous Galerkin method, multigrid iteration, two-level Fourier analysis, pointwise block-relaxation

\section{INTRODUCTION}

In this paper we describe and analyze a multigrid method for the solution of discrete systems arising from discontinuous Galerkin (DG) discretization. Since recent work by BaumannOden [13] discontinuous Galerkin discretization has become more popular for discretization of elliptic problems, in particular for application in $h p$-adaptive solvers. Originally, DG methods, based on constrained optimization [12, 6, 11], suffered from stability problems inherent in the saddle-point character of the Lagrange multiplier formulation. In $[2,14]$ these problems were overcome by introducing a penalization of the discontinuity that stabilizes the scheme. The method by Baumann-Oden modifies the saddle-point character of the problem and results in a definite (but asymmetric) discretization for the Poisson problem. Because the diffusion part is often combined with a convection term, the asymmetry is generally not seen as a disadvantage in practice. For a comprehensive survey of recent variants of DG methods and their properties we refer to [3].

In the present analysis we restrict ourselves to the Baumann-Oden and the symmetric method. Looking for an optimal efficient solution procedure that can also be applied conve- 
niently in an $h p$-adaptive context, we are led to the solution of DG discretization methods by a multigrid (MG) technique. To our knowledge, the first paper on multigrid in combination with DG was [7], who give an abstract convergence theorem for the symmetric case along the lines of [5]. In [4] the use of MG with DG for application in groundwater flow by means of ILU-decomposition is mentioned. However, here no analysis was given.

In this paper we analyze not only the symmetric but also the Baumann-Oden discretization for the two-dimensional Poisson equation and we derive two-level convergence rates by local mode Fourier analysis. An analysis for the one-dimensional case was given in $[9,10]$. In this paper we show that multigrid can be a an $\mathcal{O}(N)$ solver indeed, provided that the right block-relaxation methods are used. The block-relaxation should not be based on grouping the degrees of freedom according to their cells, but -for a suitably selected polynomial baseon a grouping of degrees of freedom that can be associated with cell vertexes. Both for the Baumann-Oden and for the symmetric DG method, this strategy leads to an efficient MG method.

For the treatment of solution methods for systems arising from DG discretization of arbitrary high order, it is sufficient to study the fourth-order case, where the solution is approximated by cubics. With tensor-product piecewise cubics on each rectangular cell, the trace of a function and its normal derivative can be approximated sufficiently well on each cell boundary segment to determine the fourth order discrete DG operator. All additional accuracy can be obtained by corrections on a hierarchical basis, which have vanishing values and normal derivatives at cell edges. This implies that all additional accuracy can be achieved by local bubble functions only, that have no contribution to cell interaction. Hence, these additional degrees of freedom are completely restricted to cell interiors and can be solved by a combination of static condensation and defect correction iteration. This is the motivation why we study here only the cubic case, and why we introduce the basis (2.6), or a variant (see also [10]) with the Jacobi polynomials $P_{n}^{(4,4)}(x)$, that satisfy an $L_{2}$-orthogonality condition on the cell interior [1, p.774].

The outline of this paper is as follows. First, in Section 2, we describe the variational form of the PDE that is the basis for our DG discretizations, and we describe the discretization stencils that occur for the discretization of the Poisson equation. As a preparation for the MG-method we also describe the stencils for the grid-transfer-operators. In Section 3 we treat the Fourier analysis tools used for the systems of grid functions that correspond with DG discretization with piecewise cubics. In Section 4 we first treat the smoothing analysis. We show that block-relaxation is stable on a reduced basis of cubic polynomials, whereas the straightforward tensor product representation is not. In Section 5 we treat the two-level analysis and we compute optimal damping parameters for the smoothing, and the spectral radii for the two-level methods, with block-Jacobi, block-Gauss-Seidel or symmetric blockGauss-Seidel relaxation.

In the last section we show by a numerical example that the actual convergence rates correspond very well with those derived by Fourier analysis. Both for the Baumann-Oden and for the symmetric DG method, this strategy leads to an $h$-independent convergence rate of at least $0.4-0.6$ per two-level cycle. 


\section{The Discontinuous Galerkin Discretization}

2.1 The weak form of the Discontinuous Galerkin Method

In order to describe the two-dimensional discretization method studied in this paper, we first give the special weak formulation of the equation. This formulation is used for the family of Discontinuous Galerkin (DG) discretization methods applied to elliptic problems [3, 10]. To continue, we consider Poisson's equation on a unit cube $\Omega \subset \mathbb{R}^{d}$, partly with Neumann and partly with Dirichlet boundary conditions:

$$
-\Delta u=f \quad \text { on } \Omega,
$$

where $u=u_{0}$ on $\Gamma_{D}$ and $\mathbf{n} \cdot \nabla u=g$ on $\Gamma_{N}$. Further we have $\Gamma_{D} \cap \Gamma_{N}=\emptyset$ and $\bar{\Gamma}_{D} \cup \bar{\Gamma}_{N}=\partial \Omega$. The variational form of this equation, for the different DG-methods reads: find $u \in H^{1}\left(\Omega_{h}\right)$ such that:

$$
B(u, v)=L(v) \quad \forall v \in H^{1}\left(\Omega_{h}\right),
$$

where the bilinear form $B(u, v)$ is defined by,

$$
\begin{aligned}
B(u, v) & =\sum_{\Omega_{e} \in \Omega_{h}} \int_{\Omega_{e}} \nabla u \cdot \nabla v d x-\int_{\Gamma_{\mathrm{int}} \cup \Gamma_{\mathrm{D}}}\langle\nabla u\rangle \cdot[v] d s \\
& +\sigma \int_{\Gamma_{\mathrm{int}} \cup \Gamma_{\mathrm{D}}}\langle\nabla v\rangle \cdot[u] d s+\mu \int_{\Gamma_{\mathrm{int}} \cup \Gamma_{\mathrm{D}}}[u] \cdot[v] d s,
\end{aligned}
$$

and the functional $L(v)$ by,

$$
L(v)=\sum_{\Omega_{e} \in \Omega_{h}} \int_{\Omega_{e}} f v d x+\sigma \int_{\Gamma_{\mathrm{D}}}\langle\nabla v\rangle \cdot\left[u_{0}\right] d s+\int_{\Gamma_{N}} g v d s .
$$

For non-negative integer $k$, the space $H^{k}\left(\Omega_{h}\right)$ is the broken Sobolev space [13] on the partitioning $\Omega_{h}$ of the domain $\Omega$,

$$
\Omega_{h}=\left\{\Omega_{e} \mid \cup_{e} \bar{\Omega}_{e}=\bar{\Omega}, \Omega_{i} \cap \Omega_{j}=\emptyset, i \neq j\right\} .
$$

The interior boundaries are denoted by $\Gamma_{\text {int }}=\cup_{e} \partial \Omega_{e} \backslash \partial \Omega$. The penalty parameter $\mu$ and method parameter $\sigma$ determine possible different discretizations [3] : $\sigma=1$ gives Baumann's method (or NIPG if $\mu>0$ ), $\sigma=-1$ gives the symmetric DG (IP-DG for $\mu>0$ ). For a scalar function $w(x)$, the jump operator $[\cdot]$ and the average operator $\langle\cdot\rangle$ are defined at the common interface ${ }^{1}$ between two cells $\Gamma_{i, j}=\bar{\Omega}_{i} \cup \bar{\Omega}_{j}$, by

$$
\begin{aligned}
{[w(x)] } & =\left.w(x)\right|_{\partial \Omega_{i}} \mathbf{n}_{i}+\left.w(x)\right|_{\partial \Omega_{j}} \mathbf{n}_{j}, \\
\langle w(x)\rangle & =\frac{1}{2}\left(\left.w(x)\right|_{\partial \Omega_{i}}+\left.w(x)\right|_{\partial \Omega_{j}}\right),
\end{aligned}
$$

for $x \in \Gamma_{i, j}$. Here $\mathbf{n}_{i}$ is the unit outward pointing normal for cell $\Omega_{i}$. For a vector valued function, $\tau(x)$, we define

$$
\begin{aligned}
{[\tau(x)] } & =\left.\tau(x)\right|_{\partial \Omega_{i}} \cdot \mathbf{n}_{i}+\left.\tau(x)\right|_{\partial \Omega_{j}} \cdot \mathbf{n}_{j}, \\
\langle\tau(x)\rangle & =\frac{1}{2}\left(\left.\tau(x)\right|_{\partial \Omega_{i}}+\left.\tau(x)\right|_{\partial \Omega_{j}}\right) .
\end{aligned}
$$

\footnotetext{
${ }^{1}$ At a Dirichlet boundary the interface with a virtual (flat, exterior) adjacent cell, containing only the Dirichlet data, is used.
} 
2.2 The discrete weak formulation in tensor product form

The next step is to define the finite dimensional test and trial function spaces, $S_{h}, V_{h} \subset$ $H^{1}\left(\Omega_{h}\right)$ in order to derive a discrete version of the weak formulation (2.1). To simplify the analysis we restrict ourselves to the two-dimensional Poisson's equation on a regular uniform partitioning. The treatment of the three-dimensional equation is analogous, but less convenient considering the notation.

Taking the same space for the test and trial functions $\left(S_{h}=V_{h}\right)$, we have:

find $u_{h} \in S_{h}$ such that,

$$
B\left(u_{h}, v_{h}\right)=L\left(v_{h}\right), \quad \forall v_{h} \in S_{h} .
$$

We take for the finite dimensional trial and test space $S_{h} \subset H^{1}\left(\Omega_{h}\right)$ the space of piecewise polynomials of degree less than $2 p$ in each of the coordinate directions on the partitioning $\Omega_{h}$ :

$$
S_{h}=\left\{\phi_{i, e} \in P^{2 p-1}\left(\Omega_{e}\right), \quad \Omega_{e} \in \Omega_{h}\right\},
$$

and, as motivated in [10], we further provide $S_{h}$ with a tensor product basis of polynomials, defined on the unit interval:

$$
\phi_{2 n+k}(t)=t^{n+k}(1-t)^{n+1-k}, \quad n=0,1, \cdots, p-1, \quad k=0,1 .
$$

Thus, on the unit cube, $\hat{\Omega} \subset \mathbb{R}^{2}$, we use a basis of tensor-product polynomials based on (2.6). A basis for $P^{2 p-1}\left(\Omega_{e}\right)$ is obtained by the usual affine mapping $\widehat{\Omega} \rightarrow \Omega_{e}$. Hence, on a regular rectangular grid $\Omega_{h}$ with cells $\Omega_{e}$ of size $h_{x} \times h_{y}$ the approximate solution reads:

$$
\begin{aligned}
u_{h}(x, y) & =\sum_{1 \leq e \leq N} \sum_{0 \leq i, j<2 p} c_{e, i, j} \phi_{i}\left(\frac{x-x_{e}}{h_{x}}\right) \phi_{j}\left(\frac{y-y_{e}}{h_{y}}\right) \\
& \equiv \sum_{1 \leq e \leq N} \sum_{0 \leq i, j<2 p} c_{e, i, j} \phi_{e, i}(\xi) \phi_{e, j}(\eta) .
\end{aligned}
$$

After substitution of (2.7) into (2.5) and because of the tensor product structure of our basis, we can express the discrete system $L_{h} u_{h}=f_{h}$ in explicit form as:

$$
\begin{gathered}
\sum_{1 \leq e \leq N} \sum_{0 \leq i, j<2 p} c_{e, i, j}\left[\left(\frac{1}{h_{x}} \int_{0}^{1} \phi_{e, i}^{\prime} \phi_{e, \tilde{i}}^{\prime} d \xi-\left.\frac{1}{h_{x}}\left\langle\nabla \phi_{e, i}\right\rangle \cdot\left[\phi_{e, \tilde{i}}\right]\right|_{\Gamma_{\mathrm{int}} \cup \Gamma_{\mathrm{D}}}+\right.\right. \\
\left.\left.\sigma \frac{1}{h_{x}}\left[\phi_{e, i}\right] \cdot\left\langle\nabla \phi_{e, \tilde{i}}\right\rangle\right|_{\Gamma_{\mathrm{int}} \cup \Gamma_{\mathrm{D}}}+\left.\mu\left[\phi_{e, i}\right] \cdot\left[\phi_{e, \tilde{i}}\right]\right|_{\Gamma_{\mathrm{int}} \cup \Gamma_{\mathrm{D}}}\right) h_{y} \int_{0}^{1} \phi_{e, j} \phi_{e, \tilde{j}} d \eta \\
+h_{x} \int_{0}^{1} \phi_{e, i} \phi_{e, \tilde{i}} d \xi\left(\frac{1}{h_{y}} \int_{0}^{1} \phi_{e, j}^{\prime} \phi_{e, \tilde{j}}^{\prime} d \eta-\left.\frac{1}{h_{y}}\left\langle\nabla \phi_{e, j}\right\rangle \cdot\left[\phi_{e, \tilde{j}}\right]\right|_{\Gamma_{\mathrm{int}} \cup \Gamma_{\mathrm{D}}+}\right. \\
\left.\left.\left.\quad \sigma \frac{1}{h_{y}}\left[\phi_{e, j}\right] \cdot\left\langle\nabla \phi_{e, \tilde{j}}\right\rangle\right|_{\Gamma_{\mathrm{int}} \cup \Gamma_{\mathrm{D}}}+\left.\mu\left[\phi_{e, j}\right] \cdot\left[\phi_{e, \tilde{j}}\right]\right|_{\Gamma_{\mathrm{int}} \cup \Gamma_{\mathrm{D}}}\right)\right] \\
=\sum_{1 \leq e \leq N} \sum_{0 \leq i, j<2 p} \int_{\Omega_{e}} f(x, y) \phi_{\tilde{i}}\left(\frac{x-x_{e}}{h_{x}}\right) \phi_{\tilde{j}}\left(\frac{y-y_{e}}{h_{y}}\right) d \Omega_{e}+\sigma \int_{\Gamma_{D}}\left\langle\nabla\left(\phi_{\tilde{i}}\left(\frac{x-x_{e}}{h_{x}}\right) \phi_{\tilde{j}}\left(\frac{y-y_{e}}{h_{y}}\right)\right)\right\rangle \cdot\left[u_{0}\right] d s \\
+\mu \int_{\Gamma_{D}}\left[u_{0}\right] \cdot\left[\phi_{\tilde{i}}\left(\frac{x-x_{e}}{h_{x}}\right) \phi_{\tilde{j}}\left(\frac{y-y_{e}}{h_{y}}\right)\right] d s \int_{\Gamma_{N}} g \phi_{\tilde{i}}\left(\frac{x-x_{e}}{h_{x}}\right) \phi_{\tilde{j}}\left(\frac{y-y_{e}}{h_{y}}\right) d s, \quad \forall \tilde{i}, \tilde{j} .
\end{gathered}
$$


We see that the left-hand side of (2.8) is an extension of a one-dimensional stiffness and mass matrix. If we define

$$
\mathbf{M}=\left(\mathbf{M}_{e, i, \tilde{i}}\right)=h_{x} \int_{0}^{1} \phi_{e, i} \phi_{e, \tilde{i}} d \xi
$$

and

$$
\begin{aligned}
\mathbf{S}=\left(\mathbf{S}_{e, i, \tilde{i}}\right)= & \frac{1}{h_{x}} \int_{0}^{1} \phi_{e, i}^{\prime} \phi_{e, \tilde{i}}^{\prime} d \xi-\frac{1}{h_{x}}\left\langle\nabla \phi_{e, i}\right\rangle \cdot\left[\phi_{e, \tilde{i}}\right] \mid \Gamma_{\Gamma_{\mathrm{int}} \cup \Gamma_{\mathrm{D}}}+ \\
& \left.\sigma \frac{1}{h_{x}}\left[\phi_{e, i}\right] \cdot\left\langle\nabla \phi_{e, \tilde{i}}\right\rangle\right|_{\Gamma_{\mathrm{int}} \cup \Gamma_{\mathrm{D}}}+\left.\mu\left[\phi_{e, i}\right] \cdot\left[\phi_{e, \tilde{i}}\right]\right|_{\Gamma_{\mathrm{int}} \cup \Gamma_{\mathrm{D}}},
\end{aligned}
$$

we may write:

$$
L_{h} u_{h}=\sum_{1 \leq e \leq N} \sum_{0 \leq i, j<2 p} c_{e, i, j}\left(\mathbf{S}_{e, i, \tilde{i}} \mathbf{M}_{e, j, \tilde{j}}+\mathbf{M}_{e, i, \tilde{i}} \mathbf{S}_{e, j, \tilde{j}}\right), \quad \forall \tilde{i}, \tilde{j}
$$

Or briefly, in tensor product notation we have:

$$
L_{h} u_{h}=\sum_{1 \leq e \leq N} \sum_{0 \leq i, j<2 p} c_{e, i, j}(\mathbf{S} \otimes \mathbf{M}+\mathbf{M} \otimes \mathbf{S})_{e, i, \tilde{i}, j, \tilde{j}}, \quad \forall \tilde{i}, \tilde{j}
$$

In our one-dimensional analysis $[9,10]$ we explained that if we associate the first four polynomials of basis (2.6) with function values and corrections on derivatives at the cell corners, the discrete system can be partitioned in point-wise blocks, each of which can be associated with a nodal point of a cell. We showed by Fourier analysis, that the relaxation methods (damped block Gauss Seidel (DGS) and damped block Jacobi (JOR)), based on that partitioning, have better smoothing properties than the classical cell-wise partitioning. We further emphasized that higher order polynomials can be introduced as genuine bubble functions. They correspond to interior cell corrections only. So, if we are interested in fast convergence of the discrete system the coefficients of these bubble functions are of less importance. They can be eliminated by static condensation or dealt with defect correction. So, in this two-dimensional analysis, we again restrict ourselves to the case $p=2$ and we distinguish between cell-wise and point-wise stencils of the discrete system.

\subsection{The two-dimensional cell-wise and point-wise stencil}

Whereas for the one-dimensional discrete system the point-wise and the cell-wise stencil are both three-point block stencils, this is not the case for the two dimensional discrete system described above. To see this, consider (2.8) and (2.9). If we order the equations and coefficients of the stiffness and mass matrices cell-wise over the two coordinate directions $\left(\left[\phi_{e, 0}(),. \phi_{e, 2}(),. \phi_{e, 3}(),. \phi_{e, 1}().\right]\right)$, we have the following stencil contributions:

$$
\mathbf{S}_{L}^{C}=\left[\begin{array}{cccc}
-\frac{1}{2} & 0 & -\frac{1}{2} & \frac{1-\sigma}{2}-h \mu \\
0 & 0 & 0 & \frac{1}{2} \sigma \\
0 & 0 & 0 & 0 \\
0 & 0 & 0 & \frac{1}{2} \sigma
\end{array}\right], \mathbf{S}_{C}^{C}=\left[\begin{array}{cccc}
\frac{1+\sigma}{2}+h \mu & \frac{1}{2} & 0 & \frac{-1-\sigma}{2} \\
-\frac{1}{2} \sigma & \frac{1}{15} & \frac{1}{30} & 0 \\
0 & \frac{1}{30} & \frac{1}{2} \sigma \\
\frac{-1-\sigma}{2} & 0 & \frac{1}{2} & \frac{1+\sigma}{2}+h \mu
\end{array}\right]
$$




$$
\mathbf{S}_{R}^{C}=\left[\begin{array}{cccc}
\frac{1}{2} \sigma & 0 & 0 & 0 \\
0 & 0 & 0 & 0 \\
\frac{1}{2} \sigma & 0 & 0 & 0 \\
\frac{1-\sigma}{2}-h \mu & -\frac{1}{2} & 0 & -\frac{1}{2}
\end{array}\right], \mathbf{M}_{C}^{C}=\left[\begin{array}{cccc}
\frac{1}{3} & \frac{1}{20} & \frac{1}{30} & \frac{1}{6} \\
\frac{1}{20} & \frac{1}{105} & \frac{1}{140} & \frac{1}{30} \\
\frac{1}{10} & \frac{1}{140} & \frac{1}{105} & \frac{1}{20} \\
\frac{1}{6} & \frac{1}{30} & \frac{1}{20} & \frac{1}{3}
\end{array}\right]
$$

where the superscript 'C' denotes 'cell-wise' and the subscript 'L,C,R', respectively 'left', 'center' and 'right'. If we now according to (2.9) write:

$$
\mathbf{L}_{.}^{C}=\left(\mathbf{S}_{.}^{C} \otimes \mathbf{M}_{.}^{C}+\mathbf{M}_{.}^{C} \otimes \mathbf{S}_{.}^{C}\right)_{i, \tilde{i}, j, \tilde{j}}, \quad i, \tilde{i}, j, \tilde{j} \in\{1,2, . ., 4\},
$$

the result is a five-points block stencil, with for each block a $16 \times 16$ matrix. We denote the stencil by:

$$
L_{h} \approx \frac{\mathbf{L}_{L C}^{C}}{\frac{\mathbf{L}_{C L}^{C}}{\mathbf{L}_{C C}^{C}}} \frac{\mathbf{L}_{C R}^{C}}{\mathbf{L}_{R C}^{C}} .
$$

Re-ordering the equations and coefficients of the mass and stiffness matrices in a point-wise manner (collecting $\left[\phi_{e-1,3}, \phi_{e-1,1}, \phi_{e, 0}, \phi_{e, 2}\right]$ ) over the two coordinate directions, yields the following stencil contributions [10]:

$$
\begin{aligned}
& \mathbf{S}_{L}^{P}=\left[\begin{array}{cccc}
0 & 0 & 0 & \frac{1}{30} \\
0 & \frac{1}{2} \sigma & \frac{-1-\sigma}{2} & 0 \\
0 & 0 & -\frac{1}{2} & 0 \\
0 & 0 & 0 & 0
\end{array}\right], \mathbf{S}_{C}^{P}=\left[\begin{array}{cccc}
\frac{2}{15} & -\frac{1}{2} \sigma & \frac{1}{2} \sigma & 0 \\
\frac{1}{2} & \frac{1+\sigma}{2}+h \mu & \frac{1-\sigma}{2}-h \mu & -\frac{1}{2} \\
-\frac{1}{2} & \frac{1-\sigma}{2}-h \mu & \frac{1+\sigma}{2}+h \mu & \frac{1}{2} \\
0 & \frac{1}{2} \sigma & -\frac{1}{2} \sigma & \frac{2}{15}
\end{array}\right], \mathbf{S}_{R}^{P}=\left[\begin{array}{cccc}
0 & 0 & 0 & 0 \\
0 & -\frac{1}{2} & 0 & 0 \\
0 & \frac{-1-\sigma}{2} & \frac{1}{2} \sigma & 0 \\
\frac{1}{30} & 0 & 0 & 0
\end{array}\right] \\
& \mathbf{M}_{L}^{P}=\left[\begin{array}{cccc}
0 & 0 & \frac{1}{30} & \frac{1}{140} \\
0 & 0 & \frac{1}{6} & \frac{1}{30} \\
0 & 0 & 0 & 0 \\
0 & 0 & 0 & 0
\end{array}\right], \mathbf{M}_{C}^{P}=\left[\begin{array}{cccc}
\frac{1}{105} & \frac{1}{20} & 0 & 0 \\
\frac{1}{20} & \frac{1}{3} & 0 & 0 \\
0 & 0 & \frac{1}{3} & \frac{1}{20} \\
0 & 0 & \frac{1}{20} & \frac{1}{105}
\end{array}\right], \mathbf{M}_{R}^{P}=\left[\begin{array}{cccc}
0 & 0 & 0 & 0 \\
0 & 0 & 0 & 0 \\
\frac{1}{30} & \frac{1}{6} & 0 & 0 \\
\frac{1}{140} & \frac{1}{30} & 0 & 0
\end{array}\right],
\end{aligned}
$$

where the superscript 'P' stands for 'point-wise'. Then evaluation of (2.10) now yields the nine-points block stencil:

$$
L_{h} \approx \begin{array}{|c|c|c|}
\hline \mathbf{L}_{L L}^{P} & \mathbf{L}_{C L}^{P} & \mathbf{L}_{R L}^{P} \\
\hline \mathbf{L}_{L C}^{P} & \mathbf{L}_{C C}^{P} & \mathbf{L}_{R C}^{P} \\
\hline \mathbf{L}_{L R}^{P} & \mathbf{L}_{C R}^{P} & \mathbf{L}_{R R}^{P} \\
\hline
\end{array}
$$

Every block is a $16 \times 16$ matrix containing information about the 4 cells around the point in the computational domain. The cell-wise and point-wise stencils represent the same discretization. The different ordering, only results in different relaxation behavior of the blockrelaxation procedures.

\subsection{Restrictions and Prolongations}

As we are interested in multigrid solution methods we have to define restrictions and prolongations. In [10] we derived the natural prolongation, the injective restriction and the Galerkin restriction operator for the one-dimensional polynomial basis. We further stated that extension to higher dimensions is easily made by means of the tensor product principle. However, for convenience we give in this section an overview of the conclusions.

For the two-dimensional analysis, we consider a uniform fine partitioning of cells $\Omega_{h}$ with size $h_{1} \times h_{2}$ and a uniform coarse cell partitioning $\Omega_{H}$ of cells $H_{1} \times H_{2}=2 h_{1} \times 2 h_{2}$. With $j h=\left(j_{1} h_{1}, j_{2} h_{2}\right)$ and $j_{H}=\left(j_{1} H_{1}, j_{2} H_{2}\right)$, we denote the nodal points of respectively 
the fine and coarse partitioning. We further denote the spaces of discontinuous piecewise polynomials by $S_{h}$ and $S_{H}$. Since, by nesting we have $S_{H} \subset S_{h}$, the natural prolongation $P_{h H}: S_{H} \rightarrow S_{h}$ is defined ${ }^{2}$ such that $\left(P_{h H} u_{H}\right)(x)=u_{H}(x)$ for all $x \in\left(\mathbb{R} \backslash \mathbb{Z}_{h}\right)^{2}$. For our piecewise cubics $(p=2)$ the one-dimensional prolongation stencil $\left[\mathbf{P}_{L L}, \mathbf{P}_{L} \mathbf{P}_{C}, \mathbf{P}_{R} \mathbf{P}_{R R}\right]$ reads (see $[10]$ ):

$$
\begin{aligned}
\mathbf{P}_{L L} & =\left[\begin{array}{cccc}
0 & 0 & 0 & -\frac{1}{8} \\
0 & 0 & 0 & 0 \\
0 & 0 & 0 & 0 \\
0 & 0 & 0 & 0
\end{array}\right], \mathbf{P}_{L}=\left[\begin{array}{cccc}
0 & 0 & 0 & \frac{1}{4} \\
0 & 0 & \frac{1}{2} & \frac{4}{8} \\
0 & 0 & \frac{1}{2} & \frac{1}{8} \\
0 & 0 & 0 & 0
\end{array}\right], \mathbf{P}_{C}=\left[\begin{array}{cccc}
\frac{3}{8} & 0 & 0 & 0 \\
0 & 1 & 0 & 0 \\
0 & 0 & 1 & 0 \\
0 & 0 & 0 & \frac{3}{8}
\end{array}\right], \\
& \mathbf{P}_{R}=\left[\begin{array}{cccc}
0 & 0 & 0 & 0 \\
\frac{1}{1} & \frac{1}{1} & 0 & 0 \\
\frac{9}{8} & \frac{1}{2} & 0 & 0 \\
\frac{1}{4} & 0 & 0 & 0
\end{array}\right], \mathbf{P}_{R R}=\left[\begin{array}{cccc}
0 & 0 & 0 & 0 \\
0 & 0 & 0 & 0 \\
0 & 0 & 0 & 0 \\
-\frac{1}{8} & 0 & 0 & 0
\end{array}\right] .
\end{aligned}
$$

Then, we derive the two-dimensional prolongation stencil from (cf. (2.9)):

$$
P_{h H} \approx(\mathbf{P} . \otimes \mathbf{P} .)_{i, \tilde{i}, j, \tilde{j}} \quad i, \tilde{i}, j, \tilde{j} \in\{1,2, . ., 4\},
$$

where the dot-subscript (LL, L,C,...) denotes 'outer-left, left, center' etc. The result is a 25points block-stencil, with each block a $16 \times 16$ matrix, associated with a neighboring nodal point.

Whereas the prolongation $P_{h H}$ is uniquely defined, the restriction operator is not. However, we recognize two natural restriction operators. The first one is the restriction for the residual, characterized as the Galerkin restriction. Due to the weighed residual character of the Galerkin discretization, this restriction operator is the adjoint of the prolongation: $\bar{R}_{H h}=\left(P_{h H}\right)^{T}$. The Toeplitz operator of the Galerkin restriction is the transpose of the Toeplitz operator for the prolongation. Because of the Galerkin construction of the discretization and the nesting of the spaces $S_{H}$ and $S_{h}$, the Galerkin relation holds for the discretization on the coarser and finer grid:

$$
L_{H}=\bar{R}_{H h} L_{h} P_{h H} .
$$

The stencil representation of $\bar{R}_{H h}$ is the same as for $P_{h H}$.

The second natural restriction is the injective restriction, applied in the solution space. This restriction is based on function values and corrections on derivatives at the cell corners [10]. Because of our basis (2.6), the one-dimensional restriction operator is constructed such that:

$$
\begin{aligned}
\left.(d / d x)\left(R_{H h} u_{h}\right)(j H)\right|_{\Omega_{H, j-1}} & =\left.(d / d x) u_{h}(2 j h)\right|_{\Omega_{h, 2 j-1}}, \\
\left.\left(R_{H h} u_{h}\right)(j H)\right|_{\Omega_{H, j-1}} & =\left.u_{h}(2 j h)\right|_{\Omega_{h, 2 j-1}}, \\
\left.\left(R_{H h} u_{h}\right)(j H)\right|_{\Omega_{H, j}} & =\left.u_{h}(2 j h)\right|_{\Omega_{h, 2 j}}, \\
\left.(d / d x)\left(R_{H h} u_{h}\right)(j H)\right|_{\Omega_{H, j}} & =\left.(d / d x) u_{h}(2 j h)\right|_{\Omega_{h, 2 j}},
\end{aligned}
$$

yielding the block-stencil $\left[\mathbf{R}_{L L}, \mathbf{R}_{L} \mathbf{R}_{C}, \mathbf{R}_{R} \mathbf{R}_{R R}\right]$ :

$$
\mathbf{R}_{L L}=\left[\begin{array}{cccc}
0 & 0 & 0 & 1 \\
0 & 0 & 0 & 0 \\
0 & 0 & 0 & 0 \\
0 & 0 & 0 & 0
\end{array}\right], \mathbf{R}_{L}=\left[\begin{array}{lll}
0 & & \\
& & \\
& & 0
\end{array}\right], \mathbf{R}_{C}=\left[\begin{array}{cccc}
3 & 0 & 0 & 0 \\
0 & 1 & 0 & 0 \\
0 & 0 & 1 & 0 \\
0 & 0 & 0 & 3
\end{array}\right],
$$

\footnotetext{
${ }^{2} \mathbb{Z}_{h}^{2}$ is the infinite regular two-dimensional grid, defined by $\mathbb{Z}_{h}^{2}=\left\{j h \mid j \in \mathbb{Z}^{2}, h>0\right\}$, where $j h=$ $\left(j_{1} h_{1}, j_{2} h_{2}\right)$
} 


$$
\mathbf{R}_{R}=\left[\begin{array}{lll}
0 & & \\
& \cdot & \\
& & \\
& & 0
\end{array}\right], \mathbf{R}_{R R}=\left[\begin{array}{cccc}
0 & 0 & 0 & 0 \\
0 & 0 & 0 & 0 \\
0 & 0 & 0 & 0 \\
1 & 0 & 0 & 0
\end{array}\right] .
$$

This operator $R_{H h}$ is the left-inverse of $P_{h H}$, i.e. $I_{H}=R_{H h} P_{h H}$.

3. Two-dimensional Fourier analysis tools

\subsection{The Fourier transform of an n-valued two-dimensional grid function}

In [9] and [10] we have introduced some Fourier analysis tools in order to analyze the eigenvalue spectra of the one dimensional discrete system and its relaxation methods. In this section we extend this analysis for an higher dimensional discrete system. Therefore we define the regular two-dimensional grid $\mathbb{Z}_{h}^{2}$ as:

$$
\mathbb{Z}_{h}^{2}=\left\{j h \mid j \in \mathbb{Z}^{2}\right\}
$$

where

$$
j h=\left(j_{1} h_{1}, j_{2} h_{2}\right),
$$

and we denote $h^{2}=h_{1} \cdot h_{2}$. Further we introduce the two-dimensional torus

$$
\mathbb{T}_{h}^{2}=\left(-\pi / h_{1}, \pi / h_{1}\right] \times\left(-\pi / h_{2}, \pi / h_{2}\right] .
$$

Following [8], an $n$-valued two-dimensional grid function is denoted by $\mathbf{u}_{h} \in\left[l^{2}\left(\mathbb{Z}_{h}^{2}\right)\right]^{n}$ and is provided with the norm

$$
\left\|\mathbf{u}_{h}\right\|_{\left[l^{2}\left(\mathbb{Z}_{h}^{2}\right)\right]^{n}}^{2}=\sum_{1 \leq i \leq n}\left\|u_{h, i}\right\|_{l^{2}\left(\mathbb{Z}_{h}^{2}\right)}^{2}
$$

$l^{2}\left(\mathbb{Z}_{h}^{2}\right)$ is the Hilbert space of square summable two-dimensional complex grid-functions defined on $\mathbb{Z}_{h}^{2}$, with innerproduct

$$
\left(u_{h}, v_{h}\right)=h^{2} \sum_{j \in \mathbb{Z}^{2}} u_{h}(j h) \overline{v_{h}(j h)}
$$

The Fourier transform $\widehat{\mathbf{u}_{h}} \in\left[L^{2}\left(\mathbb{T}_{h}^{2}\right)\right]^{n}$ of $\mathbf{u}_{h} \in\left[l^{2}\left(\mathbb{Z}_{h}^{2}\right)\right]^{n}$ is the complex $n$-vector valued function $\mathbb{T}_{h}^{2} \rightarrow \mathbb{C}^{n}$, defined by:

$$
\widehat{\mathbf{u}_{h}}(\omega)=\left(\frac{h}{\sqrt{2 \pi}}\right)^{2} \sum_{j \in \mathbb{Z}^{2}} e^{-i(j h) \cdot \omega} \mathbf{u}_{h}(j h),
$$

and its inverse transform is given by:

$$
\mathbf{u}_{h}(j h)=\left(\frac{1}{\sqrt{2 \pi}}\right)^{2} \int_{\omega \in \mathbb{T}_{h}^{2}} e^{+i(j h) \cdot \omega} \widehat{\mathbf{u}_{h}}(\omega) d \omega .
$$

Furthermore we have by Parseval's equality:

$$
\left\|\mathbf{u}_{h}\right\|_{\left[l^{2}\left(\mathbb{Z}_{h}^{2}\right)\right]^{n}}=\left\|\widehat{\mathbf{u}_{h}}\right\|_{\left[L^{2}\left(\mathbb{T}_{h}^{2}\right)\right]^{n}}=\sqrt{\sum_{1 \leq i \leq n}\left\|\widehat{u}_{h, i}\right\|_{\mathbb{T}_{h}^{2}}^{2}},
$$

where $\widehat{\mathbf{u}_{h}}=\left\{\widehat{u}_{h, i}\right\}, i \in\{1,2, . ., n\}$. 
3.2 The Toeplitz operator on n-valued two-dimensional grid functions

Following the same approach as in $[9,10]$, for an infinite block operator obtained from a two-dimensional discretization we write $A_{h}:\left[l^{2}\left(\mathbb{Z}_{h}^{2}\right)\right]^{n} \rightarrow\left[l^{2}\left(\mathbb{Z}_{h}^{2}\right)\right]^{n}$, where $A_{h}=\left(\mathbf{a}_{m, j}\right)_{m, j \in \mathbb{Z}^{2}}$ with $\mathbf{a}_{m, j} \in \mathbb{R}^{n \times n}$. For a block Toeplitz operator $A_{h}$ we have by definition $\mathbf{a}_{m, j}=\mathbf{a}_{m-j}$ and its Fourier transform $\widehat{A_{h}}(\omega)$ is determined by:

$$
\begin{aligned}
& A_{h} e_{h, \omega}=\sum_{j \in \mathbb{Z}^{2}} \mathbf{a}_{m, j} e^{i(j h) \cdot \omega}=\widehat{A_{h}} e^{i(m h) \cdot \omega}, \text { hence, } \\
& \widehat{A_{h}}(\omega)=\sum_{j \in \mathbb{Z}^{2}} \mathbf{a}_{m, j} e^{i([j-m] h) \cdot \omega}=\sum_{k \in \mathbb{Z}^{2}} \mathbf{a}_{-k} e^{i(k h) \cdot \omega}=\sum_{k \in \mathbb{Z}^{2}} \mathbf{a}_{k} e^{-i(k h) \cdot \omega},
\end{aligned}
$$

for all $\omega=\left(\omega_{1}, \omega_{2}\right) \in \mathbb{T}_{h}^{2}$. Here $e_{h, \omega}=e^{i(j h) \cdot \omega}$ is an elementary mode defined on the regular infinite two-dimensional grid (3.1) and $\widehat{A_{h}}(\omega)$ is the $n \times n$ Fourier transform matrix of $A_{h}$. In the eigenvalue decomposition of $\widehat{A_{h}}$ :

$$
\widehat{A_{h}}(\omega) V_{h}(\omega)=\left(V_{h} \Lambda_{h}\right)(\omega),
$$

$V_{h}(\omega)$ is the $n \times n$ matrix of eigenvectors $\mathbf{v}(\omega)$ of $\widehat{A_{h}}(\omega)$. And with $\left(V_{h} \otimes e_{h, \omega}\right)(j h)=$ $V_{h}(\omega) e^{i(j h) \cdot \omega}$ we have:

$$
A_{h}\left(V_{h} \otimes e_{h, \omega}\right)=\widehat{A_{h}}(\omega)\left(V_{h} \otimes e_{h, \omega}\right)=\left(V_{h} \otimes e_{h, \omega}\right) \Lambda_{h}(\omega), \quad \omega \in \mathbb{T}_{h}^{2} .
$$

Hence the columns $\mathbf{v}(\omega) e_{h, \omega}$ of $V_{h} \otimes e_{h, \omega}$ are $n$-valued eigen (grid) functions of $A_{h}$, while $\Lambda_{h}(\omega)$ is the family of $n \times n$ diagonal matrices containing the eigenvalues of $A_{h}$ on its diagonal.

As an example we determine the eigenvalue spectra of the Toeplitz operators associated with the stencil (2.11) (or (2.12)) for respectively the symmetric DG-method $(\sigma=-1, \mu=0)$ and Baumann's DG-method $(\sigma=-1, \mu=0)$. Considering the cell-wise stencil (2.11), using (3.6) we write:

$$
\widehat{L_{h}}(\omega)=\mathbf{L}_{L C}^{C} e^{-\omega_{1} h_{1}}+\mathbf{L}_{R C}^{C} e^{\omega_{1} h_{1}}+\mathbf{L}_{C C}^{C}+\mathbf{L}_{C L}^{C} e^{-\omega_{2} h_{2}}+\mathbf{L}_{C R}^{C} e^{\omega_{2} h_{2}},
$$

where $\widehat{L_{h}}(\omega)$ is now a $16 \times 16$ matrix. By $(3.8), \Lambda_{h}(\omega)$ is the family of $16 \times 16$ matrices containing the set $\left\{\lambda_{j}(\omega)\right\}, j \in\{1,2, . ., 16\}$ eigenvalues of $L_{h}$ for the mode $e_{h, \omega}$. Figure 1 shows the in absolute value largest eigenvalue $\left.\operatorname{(max}_{j}\left(\left|\lambda_{j}(\omega)\right|\right)\right)$ of $(3.9)$ as function of $\omega \in \mathbb{T}_{h}^{2}$.

\subsection{Fourier analysis for prolongations and restrictions on two-dimensional $n$ vector valued} grid functions

Having introduced the Fourier analysis for prolongations and restrictions on one dimensional (four)-vector valued grid functions [10], the same Fourier analysis is easily applied on twodimensional $n$-valued grid functions. Key to the Fourier analysis of prolongations and restrictions are the flat prolongation and restriction operators. In the framework of this paper, we may define the flat-prolongation: $P_{h H}^{0}:\left[\ell^{2}\left(\mathbb{Z}_{H}^{2}\right)\right]^{n} \rightarrow\left[\ell^{2}\left(\mathbb{Z}_{h}^{2}\right)\right]^{n}$ simply by:

$$
\mathbf{u}_{h}(j h)=\left(P_{h H}^{0} \mathbf{u}_{H}\right)(j h)= \begin{cases}\mathbf{u}_{H}(H j / 2), & \text { if } j_{1} \text { and } j_{2} \text { even } \\ \mathbf{0}, & \text { if } j_{1} \text { and } j_{2} \text { odd }\end{cases}
$$




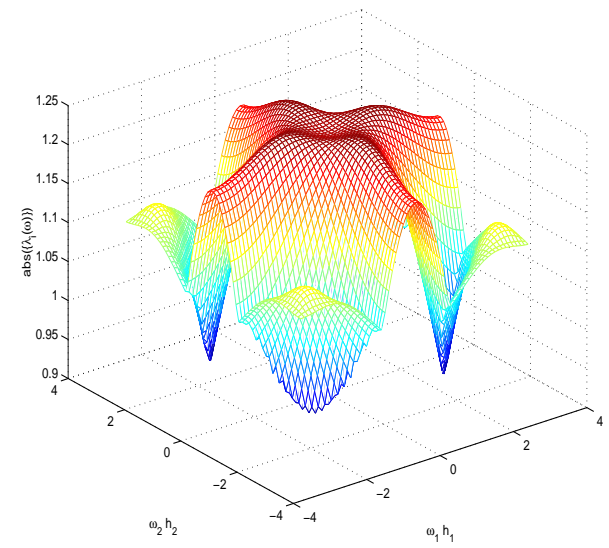

Symm-DG, $\sigma=-1, \mu=0$

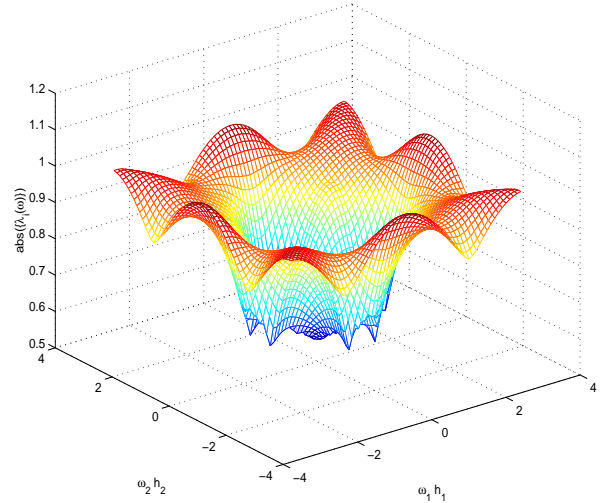

Baum-DG, $\sigma=1, \mu=0$

Figure 1: Spectral radius $\left(\max _{j}\left(\left|\lambda_{i}(\omega)\right|\right), j \in\{1,2, . ., 16\}\right)$ of $\widehat{L_{h}}(\omega)$ for the symmetric and Baumann's DG-method.

where $j$ and $h$ are multi-indices as in (3.2).

The flat-restriction: $R_{H h}^{0}:\left[\ell^{2}\left(\mathbb{Z}_{h}^{2}\right)\right]^{n} \rightarrow\left[\ell^{2}\left(\mathbb{Z}_{H}^{2}\right)\right]^{n}$ is given by:

$$
\left(R_{H h}^{0} \mathbf{u}_{h}\right)(j H)=\mathbf{u}_{h}(2 j h) .
$$

Then, according to [8] we have the relation:

$$
\widehat{P_{h H}^{0} \mathbf{u}_{H}}(\omega)=\frac{1}{4} \widehat{\mathbf{u}_{H}}(\omega), \quad \omega_{1}, \omega_{2} \in \mathbb{T}_{h}^{2},
$$

whereas the Fourier transform of the flat-restriction on a two-dimensional $n$-valued grid function is computed as:

$$
\widehat{R_{H h}^{0} \mathbf{u}_{h}}(\omega)=\sum_{p_{1}, p_{2}=0,1} \widehat{\mathbf{u}_{h}}\left(\omega_{1}+\frac{\pi p_{1}}{h_{1}}, \omega_{2}+\frac{\pi p_{2}}{h_{2}}\right), \quad \forall \omega_{1}, \omega_{2} \in T_{H}^{2}=T_{2 h}^{2} .
$$

Any constant coefficients prolongation/restriction can be constructed as a combination of a Toeplitz operator and a flat operator. And, using (3.12) the Fourier transform of a prolongation on a two-dimensional $n$-valued grid function is given by:

$$
\widehat{P_{h H} \mathbf{u}_{H}}(\omega)=\left(P_{h} \widehat{P_{h H}^{0} \mathbf{u}_{H}}\right)(\omega)=\frac{1}{4}\left[\begin{array}{l}
\widehat{P_{h}}\left(\omega_{1}, \omega_{2}\right) \\
\widehat{P_{h}}\left(\omega_{1}, \omega_{2}+\frac{\pi}{h_{2}}\right) \\
\widehat{P_{h}}\left(\omega_{1}+\frac{\pi}{h_{1}}, \omega_{2}\right) \\
\widehat{P_{h}}\left(\omega_{1}+\frac{\pi}{h_{1}}, \omega_{2}+\frac{\pi}{h_{2}}\right)
\end{array}\right] \widehat{\mathbf{u}_{h}}(\omega),
$$


$\omega_{1}, \omega_{2} \in \mathbb{T}_{H}$. For the restriction operator, using (3.13) we have:

$$
\begin{aligned}
& \widehat{R_{H h} \mathbf{u}_{h}}(\omega)=\widehat{R_{H h}^{0} R_{h}} \mathbf{u}_{h}(\omega)
\end{aligned}
$$

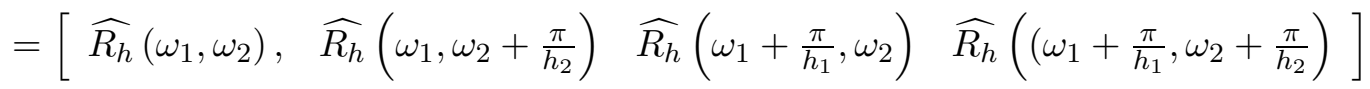

$$
\begin{aligned}
& *\left[\begin{array}{l}
\widehat{\mathbf{u}_{h}}\left(\omega_{1}, \omega_{2}\right) \\
\widehat{\mathbf{u}_{h}}\left(\omega_{1}, \omega_{2}+\frac{\pi}{h_{2}}\right) \\
\widehat{\mathbf{u}_{h}}\left(\omega_{1}+\frac{\pi}{h_{1}}, \omega_{2}\right) \\
\widehat{\mathbf{u}_{h}}\left(\omega_{1}+\frac{\pi}{h_{1}}, \omega_{2}+\frac{\pi}{h_{2}}\right)
\end{array}\right],
\end{aligned}
$$

with $\omega_{1}, \omega_{2} \in \mathbb{T}_{H}^{2}$.

\section{Two-DIMENSIONAL SMOOTHING ANALYSis}

\subsection{Smoothing analysis for the full polynomial basis}

Having introduced the Fourier analysis tools, we can study the convergence of the block relaxation methods: damped block Jacobi (JOR), damped block Gauss Seidel (DGS), and symmetric damped block Gauss Seidel, either with cell-wise or point-wise blocks. For an efficient multigrid method it is essential that the block relaxation methods show good smoothing ability. This implies that all high frequency components of the error (or residual) are damped before (or after) the approximate solution (or residual) is restricted to the coarser grid. So, for the system $A_{h} x=b$, we are interested in the convergence behavior of the iterative process:

$$
x^{(i+1)}=x^{(i)}-B_{h}\left(A_{h} x^{(i)}-b\right),
$$

where $B_{h}$ is an approximate inverse of $A_{h}$. Decomposing $A_{h}$ into a strictly block-lower, a block-diagonal and a strictly block-upper matrix,

$$
A_{h}=L+D+U,
$$

the different relaxation methods are uniquely described either by $B_{h}$ or by the amplification matrix $M_{h}^{\mathrm{REL}}=I_{h}-B_{h} A_{h}$. These operators are shown in Table 1. Because $A_{h}$ is a block-

\begin{tabular}{|c|c|c|}
\hline & $B_{h}$ & $M_{h}^{R E L}$ \\
\hline$J O R$ & $\alpha D^{-1}$ & $D^{-1}((1-\alpha) D-\alpha(L+U))$ \\
\hline$D G S_{L}$ & $\alpha(D+L)^{-1}$ & $(D+L)^{-1}((1-\alpha)(D+L)-\alpha U)$ \\
\hline$D G S_{U}$ & $\alpha(D+U)^{-1}$ & $(D+U)^{-1}((1-\alpha)(D+U)-\alpha L)$ \\
\hline \multicolumn{3}{|c|}{$\alpha>0$ is a damping parameter }
\end{tabular}

Table 1: The relaxation methods.

Toeplitz operator, also the amplification matrix $M_{h}$ is block-Toeplitz. By (3.6) and (2.11), 
we determine the Fourier transform of the different block-matrices in cell-wise ordering:

$$
\begin{aligned}
\widehat{L} & =\mathbf{L}_{C L}^{C} e^{-i \omega_{2} h_{2}}+\mathbf{L}_{L C}^{C} e^{-i \omega_{1} h_{1}}, \\
\widehat{D} & =\mathbf{L}_{C C}^{C}, \\
\widehat{U} & =\mathbf{L}_{R C}^{C} e^{i \omega_{1} h_{1}}+\mathbf{L}_{C R}^{C} e^{i \omega_{2} h_{2}},
\end{aligned}
$$

whereas the Fourier Transform of the different block-matrices in point-wise ordering yields (2.12):

$$
\begin{aligned}
\widehat{L} & =\mathbf{L}_{L L}^{P} e^{-i\left(\omega_{1} h_{1}+\omega_{2} h_{2}\right)}+\mathbf{L}_{C L}^{P} e^{-i \omega_{2} h_{2}}+\mathbf{L}_{R L}^{P} e^{i\left(\omega_{1} h_{1}-\omega_{2} h_{2}\right)}+\mathbf{L}_{L C}^{P} e^{-i \omega_{1} h_{1}}, \\
\widehat{D} & =\mathbf{L}_{C C}^{P} \\
\widehat{U} & =\mathbf{L}_{R C}^{P} e^{i \omega_{1} h_{1}}+\mathbf{L}_{L R}^{P} e^{i\left(-\omega_{1} h_{1}+\omega_{2} h_{2}\right)}+\mathbf{L}_{C R}^{P} e^{i \omega_{2} h_{2}}+\mathbf{L}_{R R}^{P} e^{i\left(\omega_{1} h_{1}+\omega_{2} h_{2}\right)} .
\end{aligned}
$$

Both cell-wise and point-wise, this yields the Fourier transform for the amplification operators for JOR, DGS and SGS:

$$
\begin{aligned}
\widehat{M_{J O R}^{R E L}} & =\widehat{D}^{-1}((1-\alpha) \widehat{D}-\alpha(\widehat{L}+\widehat{U})), \\
\widehat{M_{D G S_{L}}^{R E L}} & =(\widehat{D}+\widehat{L})^{-1}((1-\alpha)(\widehat{D}+\widehat{L})-\alpha \widehat{U}), \\
\widehat{M_{D G S_{U}}^{R E L}} & =(\widehat{D}+\widehat{U})^{-1}((1-\alpha)(\widehat{D}+\widehat{U})-\alpha \widehat{L}), \\
\widehat{M_{S G S}^{R E L}} & =\widehat{M_{D G S_{L}}^{R E L}} \widehat{M_{D G S_{U}}^{R E L}} .
\end{aligned}
$$

By (3.8) we find the eigenvalues of $M_{h}^{R E L}$ by computing the eigenvalues of $\widehat{M_{h}^{R E L}}(\omega)$ for $\omega \in \mathbb{T}_{h}^{2}$. So, both for cell-wise and point-wise relaxation methods, the Fourier transform of the amplification matrix $\widehat{M_{h}^{R E L}}(\omega)$ yields a $16 \times 16$ matrix with for each $\omega \in \mathbb{T}_{h}^{2}$ sixteen eigenvalues. The spectral radii of $\widehat{M_{h}^{R E L}}(\omega)$ for $\omega \in \mathbb{T}_{h}^{2}$ for the different relaxation methods (JOR, DGS, SGS) are shown in Table 2 for respectively the symmetric and Baumann's DGmethod. We see that, except for block Gauss Seidel applied on Baumann's DG-method,

\begin{tabular}{|c|l|l|l|l|l|l|}
\hline $\max _{\omega}|\lambda(\omega)|$ & \multicolumn{2}{|c|}{ JOR } & \multicolumn{2}{c|}{ DGS } & \multicolumn{2}{c|}{ SDGS } \\
\cline { 2 - 7 }$=\mathbb{T}_{h}^{2}$ & cell-wise & point-wise & cell-wise & point-wise & cell-wise & point-wise \\
\hline Baumann DG & 1.10 & 1.22 & 1.20 & 1.00 & 4.91 & 1.00 \\
\hline symmetric DG & - & 2.98 & - & - & - & - \\
\hline
\end{tabular}

Table 2: The in absolute value largest eigenvalue of $\widehat{M_{h}^{R E L}}(\omega)$ for the different relaxation methods for respectively the symmetric and Baumann's DG-method $(\alpha=1)$. On the empty spots in the table, $\widehat{M_{h}^{R E L}}(\omega)$ is singular.

the smoothers are unstable, or show singular behavior. Figure 2 shows the spectral radius: $\max _{j}(|\lambda(\omega)|), j \in\{1,2, . ., 16\}$ of block Gauss Seidel for Baumann's DG-method in point-wise ordering. 


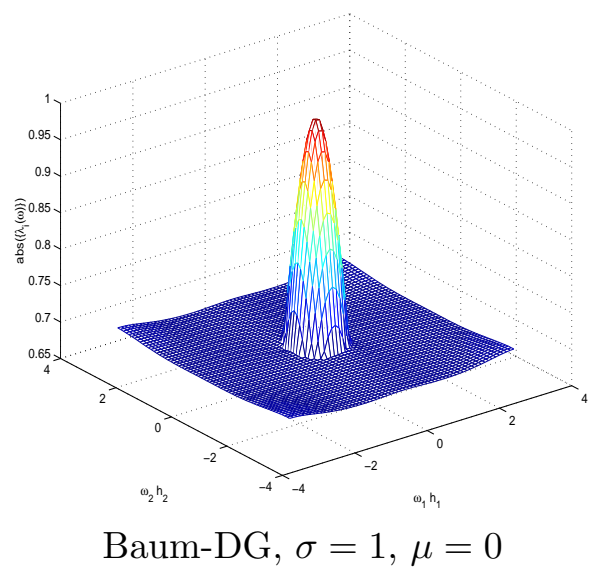

Figure 2: Spectral radius $\max _{j}\left(\left|\lambda_{j}(\omega)\right|\right), j \in\{1,2, . ., 16\}$ of $\widehat{M_{D G S_{L}}^{R E L}}(\omega)$ for Baumann's DGmethod in point-wise ordering, without damping $(\alpha=1)$.

\subsection{The reduced polynomial basis for the space $S_{h}$.}

Since we want convergence in a few iteration sweeps, we see that this block relaxation method is not suitable for multi-grid. A smoothing factor of 0.67 for $\widehat{M_{D G S_{L}}^{R E L}}$, i.e. the largest eigenvalue corresponding to the high frequencies $|\omega|>\pi / 2 h$, is not sufficiently small.

An easy heuristic explanation for the divergence of the various smoothers, is not at hand. However, an idea is that cell-wise relaxation methods mainly correct polynomial coefficients corresponding to the cell interior, while point-wise relaxation methods efficiently correct the coefficients corresponding to cell-boundaries. If we consider the twodimensional tensor product basis (2.6) for $p=2$, we associate the 16 coefficients to function values, (corrections on) $x$ and $y$ derivatives, and (corrections on) cross derivatives at the cell-corners. So we expect that this polynomial basis is suited for pointwise relaxation. However the functions associated with (corrections on) cross derivatives, $\left\{\phi_{e, 2}(x) \phi_{e, 2}(y), \phi_{e, 2}(x) \phi_{e, 3}(y), \phi_{e, 3}(x) \phi_{e, 2}(y), \phi_{e, 3}(x) \phi_{e, 3}(y)\right\}$, have small cell boundary contributions compared with the jump and flux operators in the discrete weak form (2.8). So they belong more to the class of genuine bubble functions, like the higher-order corrections in the hierarchical base. If we remove these cross-derivative contributions, we restore the typical cell boundary contribution concept of the low-order polynomial basis. We expect that the introduction of the reduced polynomial basis will lead to good smoothing properties for the point-wise relaxation methods similar as shown in $[9,10]$ for the one-dimensional case.

\subsection{The accuracy of the reduced polynomial basis}

In this section we show that the reduced polynomial basis has the same accuracy as the tensor product polynomial basis. The 12 basis-functions in the reduced basis correspond with function values and (corrections to) the $x$ - and $y$-derivatives at the two-dimensional cell corners. In the three-dimensional case the equivalent modification reduces $4^{3}=64$ functions of the tensor-product basis to a 32 -function basis representing function-values and $x-, y$ - and $z$-derivatives at the 8 corners of the three-dimensional cell. We call this basis the reduced 
polynomial basis. As mentioned in [10], the use of the reduced polynomial basis essentially reduces the amount of work for the DG-method without reducing the order of accuracy.

Considering the two-dimensional tensor product basis (2.6), for $p=2$, polynomials up to degree three in the two coordinate directions are exactly interpolated over the cells $\Omega_{e}$. So, for cells with size $h_{1} \times h_{2}$, the approximation introduces an error of

$$
\epsilon_{h}=O\left(h_{1}^{4}\right)+O\left(h_{2}^{4}\right) .
$$

Removing the basis functions $\left\{\phi_{e, 2}(x) \phi_{e, 2}(y), \phi_{e, 2}(x) \phi_{e, 3}(y), \phi_{e, 3}(x) \phi_{e, 2}(y), \phi_{e, 3}(x) \phi_{e, 3}(y)\right\}$ from the two-dimensional tensor product approximation (2.7), we lose the typical tensor product character of the approximation without affecting the order of the approximation. The removal of the basis functions responsible for the cross-derivatives introduces an extra error of order

$$
\epsilon_{\text {extra }}=O\left(h_{1}^{3}\right) O\left(h_{2}^{2}\right)+O\left(h_{1}^{2}\right) O\left(h_{2}^{3}\right) .
$$

Now, considering the total error $\epsilon_{\text {total }}=\epsilon_{h}+\epsilon_{\text {extra }}$ on a cell $\Omega_{e}$ with size $h_{1} \times h_{2}$, we distinguish the following three cases:

(1) if $h_{1}=h_{2}=h$ then: $\epsilon_{\text {total }}=O\left(h^{4}\right)+O\left(h^{3}\right) O\left(h^{2}\right)+O\left(h^{2}\right) O\left(h^{3}\right) \approx \epsilon_{h}$;

(2) if $h_{1}>h_{2}$ we find: $\epsilon_{\text {total }}=O\left(h_{1}^{4}\right) \approx \epsilon_{h}$;

(3) and if $h_{1}<h_{2}$ we get: $\epsilon_{\text {total }}=O\left(h_{2}^{4}\right) \approx \epsilon_{h}$.

So, we can remove the test- and trial functions, representing the cross-derivatives at the cell corners of a cell $\Omega_{e}$ without reducing the order of (approximation) accuracy. In the remaining of this paper we study the convergence behavior of the various smoothers for the reduced polynomial basis, which is significantly better than that for the original tensor-product basis.

\subsection{Smoothing analysis for the reduced polynomial basis}

Having reduced the polynomial basis for the test/trial space $S_{h}$, we are interested in the spectral radii of the different amplification operators $M_{h}^{R E L}$ of damped block-Jacobi, damped block-Gauss-Seidel and symmetric block-Gauss-Seidel, both in point-wise and cell-wise ordering, applied to the symmetric and Baumann's DG-method. Because of the identity (3.8) the eigenvalues $\lambda(\omega)$ of the Fourier transform $\widehat{M_{h}^{R E L}}(\omega)$ contain the eigenvalues of $M_{h}^{R E L}$. We calculate the Fourier transform $\widehat{M_{h}^{R E L}}(\omega)$ by either (4.3) or (4.4) or (4.6), now yielding a $12 \times 12$ matrix. So for every $\omega \in \mathbb{T}_{h}^{2}$ we find 12 eigenvalues. For the different relaxation methods (JOR, DGS, SGS), the spectral $\operatorname{radii}_{\max _{j}}\left(\left|\lambda_{j}(\omega)\right|\right), j=\{1,2, . ., 12\}$ of $\widehat{M_{h}^{R E L}}(\omega)$ as function of $\omega \in \mathbb{T}_{h}^{2}$, for respectively the symmetric and Baumann's DG-method are shown in the Figures 3 and 4.

The spectra of all shown relaxation methods, have an eigenvalue $|\lambda(\omega)|=1$ for $\omega_{1}=\omega_{2}=0$. This is the eigenvalue corresponding to the undamped mode, which is taken care of by the boundary conditions. The cell-wise relaxation methods cannot be applied for the symmetricDG method, because the operator $B_{h}$ is singular. However the corresponding point-wise relaxation methods are stable. For Baumann's DG-method, we see the better smoothing behavior of the point-wise relaxation methods. 

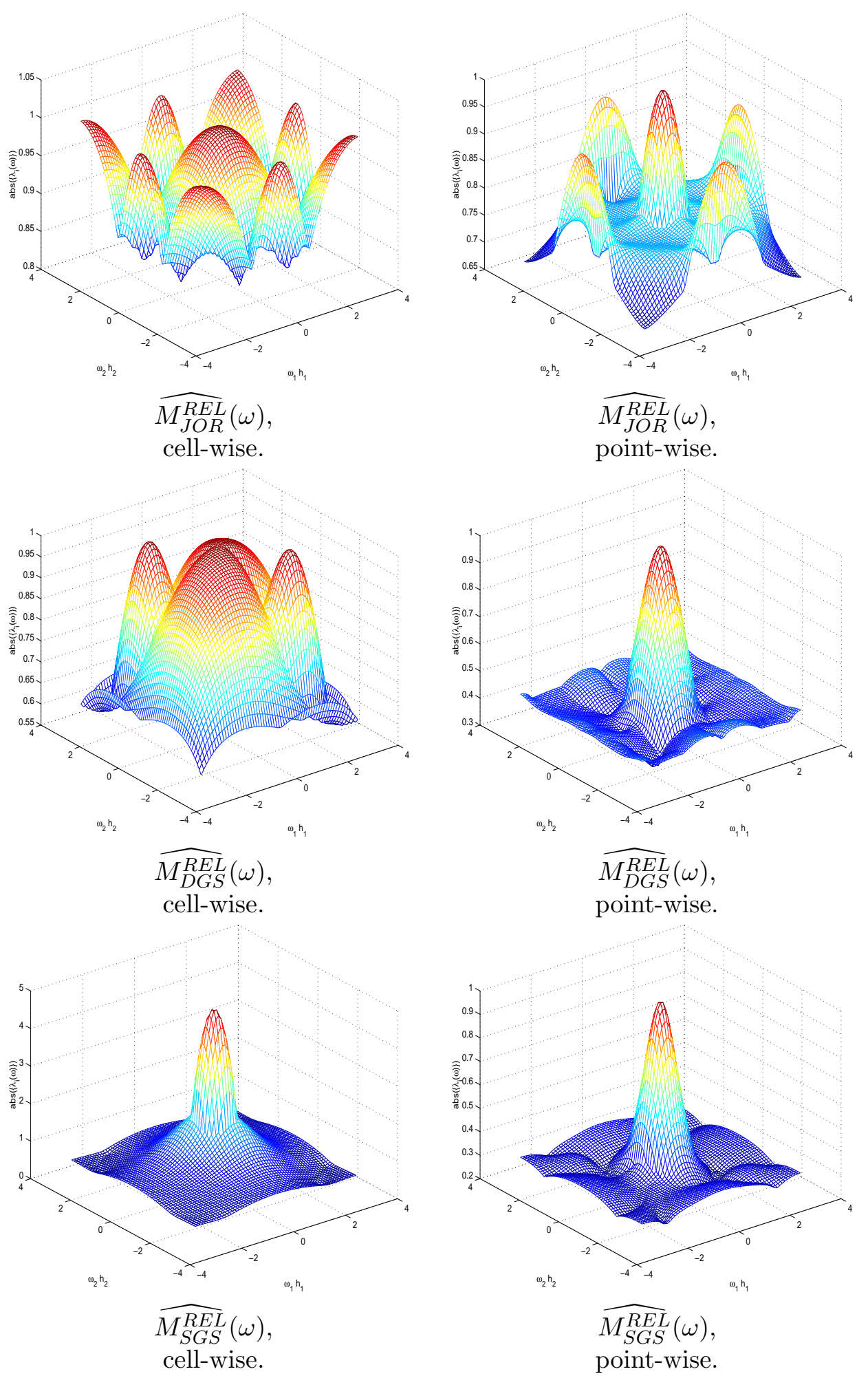

Figure 3: Spectral radii $\left(\max _{j}\left(\left|\lambda_{j}(\omega)\right|\right), j \in\{1,2, . ., 12\}\right)$ of $\widehat{M_{h}^{R E L}}(\omega)$ for Baumann's DGmethod $(\sigma=1)$ in point-wise and cell-wise ordering, without damping. 

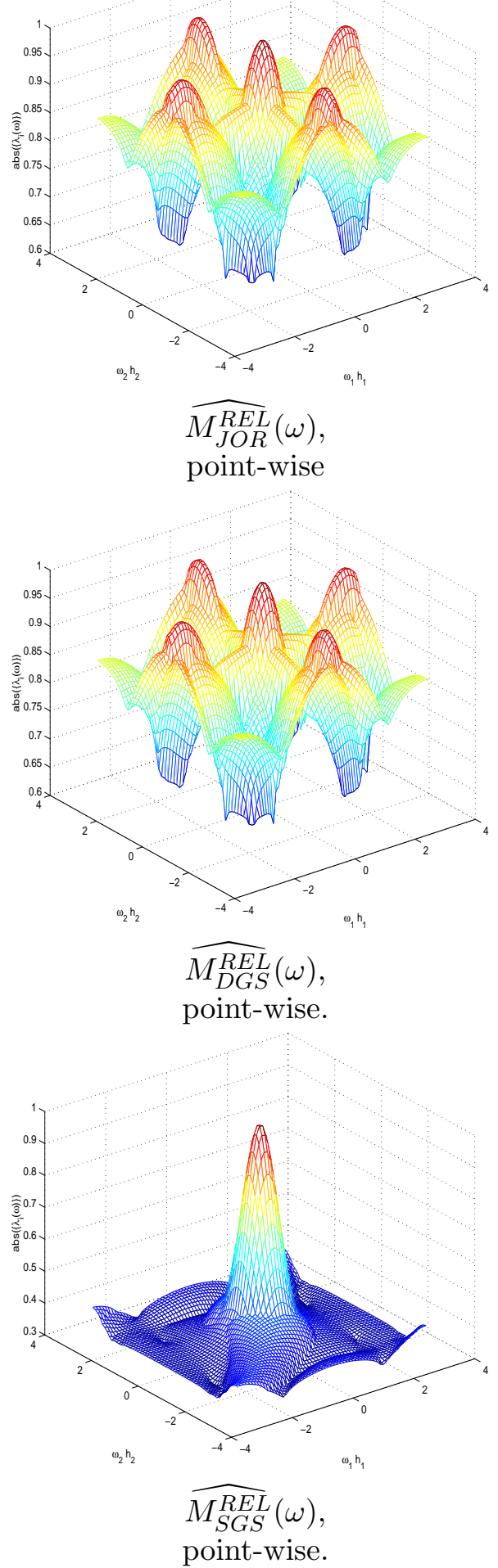

Figure 4: Spectral radii $\left(\max _{j}\left(\left|\lambda_{j}(\omega)\right|\right), j \in\{1,2, . ., 12\}\right)$ of $\widehat{M_{h}^{R E L}}(\omega)$ for the Symmetric DG-method $(\sigma=-1)$ in point-wise ordering, without damping. 


\section{TWO-LEVEL ANALYSIS}

5.1 The Fourier transform of the two-level amplification operator

Having determined the behavior of the amplification operators as a function of $\omega \in \mathbb{T}_{h}^{2}$ for the different relaxation methods, we are now interested in the convergence behavior of the two-level operator. For that, the amplification operator of the two-level algorithm for the error is given by

$$
\begin{aligned}
M_{h}^{T L A} & =\left(M_{h}^{\mathrm{REL}}\right)^{\nu_{2}} M_{h}^{\mathrm{CGC}}\left(M_{h}^{\mathrm{REL}}\right)^{\nu_{1}} \\
& =\left(M_{h}^{\mathrm{REL}}\right)^{\nu_{2}}\left(I-P_{h H} L_{H}^{-1} \bar{R}_{H h} L_{h}\right)\left(M_{h}^{\mathrm{REL}}\right)^{\nu_{1}},
\end{aligned}
$$

where $\nu_{1}$ and $\nu_{2}$ are the number of pre- (post-) relaxation sweeps respectively. $M_{h}^{C G C}$ is the amplification operator of the coarse grid correction. The amplification operator for the residue is:

$$
\begin{aligned}
\bar{M}_{h}^{T L A} & =\left(\bar{M}_{h}^{R E L}\right)^{\nu_{2}} \bar{M}_{h}^{C G C}\left(\bar{M}_{h}^{R E L}\right)^{\nu_{1}} \\
& =\left(L_{h} M_{h}^{\mathrm{REL}} L_{h}^{-1}\right)^{\nu_{2}} L_{h} M_{h}^{\mathrm{CGC}} L_{h}^{-1}\left(L_{h} M_{h}^{\mathrm{REL}} L_{h}^{-1}\right)^{\nu_{1}} .
\end{aligned}
$$

Because of the definition of the restriction (3.14) and prolongation (3.15), it follows that the Fourier transform of the the coarse grid correction $M_{h}^{C G C}$ is:

$$
\begin{aligned}
& \widehat{M_{h}^{\mathrm{CGC}}}(\omega)=\left(\widehat{I_{h}}-\widehat{P_{h H}} \widehat{L_{H}^{-1}} \widehat{R_{H h}} \widehat{L_{h}}\right)(\omega) \\
& =\left(\begin{array}{llll}
\mathbf{I} & 0 & 0 & 0 \\
0 & \mathbf{I} & 0 & 0 \\
0 & 0 & \mathbf{I} & 0 \\
0 & 0 & 0 & \mathbf{I}
\end{array}\right)-\left(\begin{array}{l}
\widehat{P_{h}}\left(\omega_{1}, \omega_{2}\right) \\
\widehat{P_{h}}\left(\omega_{1}, \omega_{2}+\pi / h_{2}\right) \\
\widehat{P_{h}}\left(\omega_{1}+\pi / h_{1}, \omega_{2}\right) \\
\widehat{P_{h}}\left(\omega_{1}+\pi / h_{1}, \omega_{2}+\pi / h_{2}\right)
\end{array}\right)\left(\widehat{L_{H}}\left(\omega_{1}, \omega_{2}\right)\right)^{-1}
\end{aligned}
$$

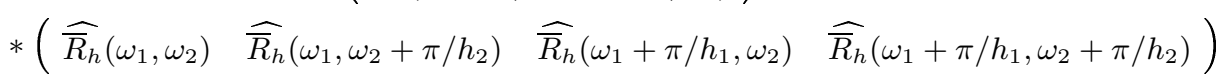

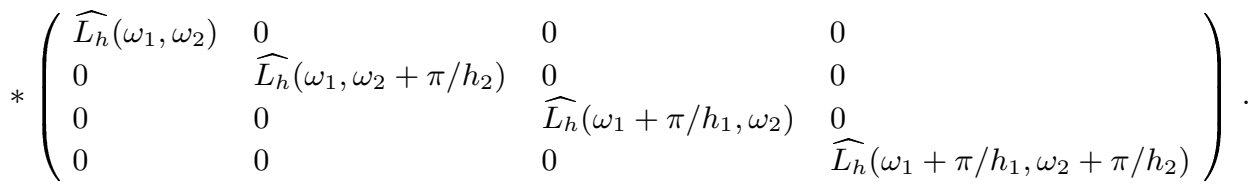

In view of the reduced polynomial basis, the Fourier transform of $\widehat{M_{h}^{\mathrm{CGC}}}(\omega)$ is an $48 \times 48$ matrix for each $\omega \in \mathbb{T}_{H}^{2}$. And because of the identity (3.8), the eigenvalues $\lambda_{i}(\omega)$ of $\widehat{M_{h}^{\mathrm{CGC}}}(\omega)$ contain the eigenvalues of $M^{\text {CGC }}$ i.e., the block-Toeplitz operator of the two-level operator. Because of the bad smoothing behavior of JOR, DGS and SDGS in cell-wise block-ordering, we abandon these block relaxation methods in the remainder of this paper and in the next section we study the point-wise smoothers and derive optimal damping factors for the twolevel algorithm.

\subsection{Optimal block-smoothing factors for the coarse grid correction}

In the local mode analysis, low and high frequency grid functions in $\left[l^{2}\left(\mathbb{Z}_{h}^{2}\right)\right]^{n}$ are usually defined as the functions that are linear combinations of modes $e_{h, \omega}=e^{i(j h) \cdot \omega}$, with respectively $\omega \in \mathbb{T}_{2 h}^{2}$ and $\omega \in \mathbb{T}_{h}^{2} \backslash \mathbb{T}_{2 h}^{2}$. However, to obtain optimal damping factors for the different relaxation methods in combination with the coarse grid correction, $M_{h}^{C G C}$, we redefine the low and high frequency grid functions. For that purpose, we consider the amplification operator of the coarse grid correction $M_{h}^{C G C}=I-P_{h H} L_{H}^{-1} \bar{R}_{H h} L_{h}$. Because of the Galerkin relation 
(2.14), $P_{h H} L_{H}^{-1} \bar{R}_{H h} L_{h}$ is a projection operator and we define low frequency components in the error as those components that lie in the range of $P_{h H} L_{H}^{-1} \bar{R}_{H h} L_{h}$. Then the high frequency components are those in the range of $I-P_{h H} L_{H}^{-1} \bar{R}_{H h} L_{h}$. So, for a "slowly varying" $n$-valued grid function $\mathbf{u}_{h}^{L F}$ we have:

$$
P_{h H} L_{H}^{-1} \bar{R}_{H h} L_{h} \mathbf{u}_{h}=\mathbf{u}_{h}^{L F},
$$

while for a "high frequency" grid function $\mathbf{u}_{h}^{H F}$ :

$$
\left(I-P_{h H} L_{H}^{-1} \bar{R}_{H h} L_{h}\right) \mathbf{u}_{h}=\mathbf{u}_{h}^{H F} .
$$

Since $M_{h}^{C G C} \mathbf{u}_{h}^{L F}=0$, we want the relaxation methods to optimally damp the contributions (5.5). Therefore, for the different relaxation methods, we seek damping parameters $\alpha_{\text {opt }}$ such that the spectral radius of $M_{h}^{C G C} M_{h}^{R E L}$ is minimal. Notice that according to (5.1), $M_{h}^{C G C} M_{h}^{R E L}$ is just the two-level operator on the error $M_{h}^{T L A}$ with $\nu_{1}=1, \nu_{2}=0$.

By (5.3) and by either (4.3), (4.4) or (4.6), we compute the eigenvalue spectra, of $M_{h}^{C G C} M_{h}^{R E L}$, first without damping $(\alpha=1)$. We determine the optimal damping parameter for the relaxation by:

$$
\alpha_{o p t}=\frac{2}{2-\left(\lambda_{\min }+\lambda_{\max }\right)},
$$

where $\lambda_{\min }$ and $\lambda_{\max }$ are respectively the minimum and maximum real eigenvalues of the spectrum without damping. It is clear that the spectral radius for the two-level operator on the residue is the same as that for the error: $\rho\left(M_{h}^{C G C} M_{h}^{R E L}\right)=\rho\left(\bar{M}_{h}^{R E L} \bar{M}_{h}^{C G C}\right)$.

The optimal damping parameters for the different two-level operators are given in Table 3 , the minimized spectral radii in Table 4 . The spectral radii of $\widehat{M_{h}^{C G C}}(\omega) \widehat{M_{h}^{R E L}}(\omega)$ as function of $\omega \in \mathbb{T}_{H}^{2}$, with optimal damping are shown in Figure 5.

From Table 4, we see that all two-level algorithms converge. Baumann's DG-method converges faster than the symmetric DG-method. This is also reflected in the two-norm of the amplification operator of the two-level algorithm. The Table 5 shows the two-norm of the amplification operator of the residue after respectively 1,3 and 4 iteration(s). We see that, except for block Jacobi on the symmetric DG-method, reduction of the residue is guaranteed within four iteration steps. For the symmetric DG-method, the spectral norms of the iteration operator on the error are the same as for the residue. In case of Baumann's DG-method, the norm of the error amplification operator becomes unbounded for vanishing frequency $\omega$. This phenomenon was also observed in [10] for the the error amplification norm in case of the one-dimensional Poisson's equation and is due to the adjoint inconsistency of the method [3].

\section{Numerical RESUlts}

Having determined optimal damping parameters for the two-level algorithm, we want to check the results by a numerical experiment. For that purpose, we solve the following twodimensional Poisson's equation on the unit square:

$$
-\left(u_{x x}+u_{y y}\right)=\frac{e^{x / \epsilon}+e^{y / \epsilon}}{\epsilon^{2}\left(e^{1 / \epsilon}-1\right)}, \text { in } \Omega,
$$




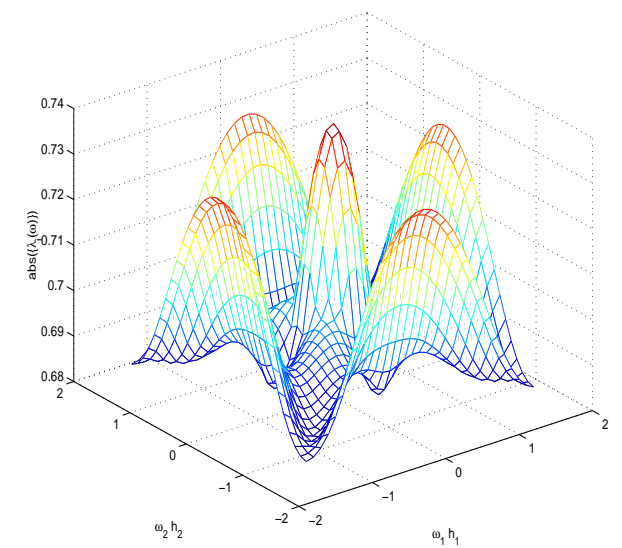

Baum-DG, $\rho\left(\widehat{M_{h}^{C G C}}(\omega) \widehat{M_{J O R}^{R E L}}(\omega)\right)$,

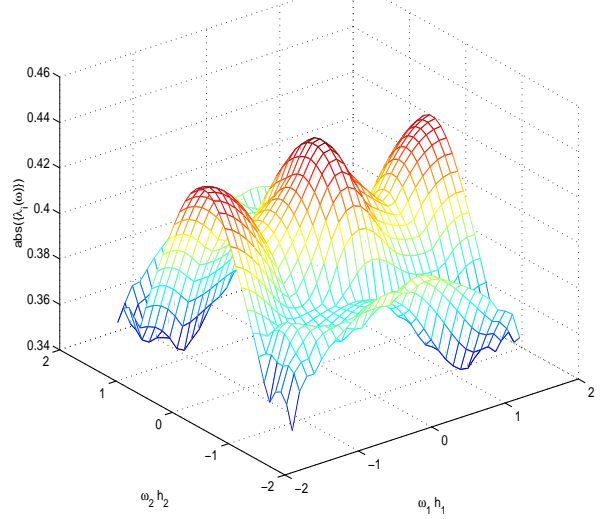

Baum-DG, $\rho\left(\widehat{M_{h}^{C G C}}(\omega) \widehat{M_{D G S}^{R E L}}(\omega)\right)$,

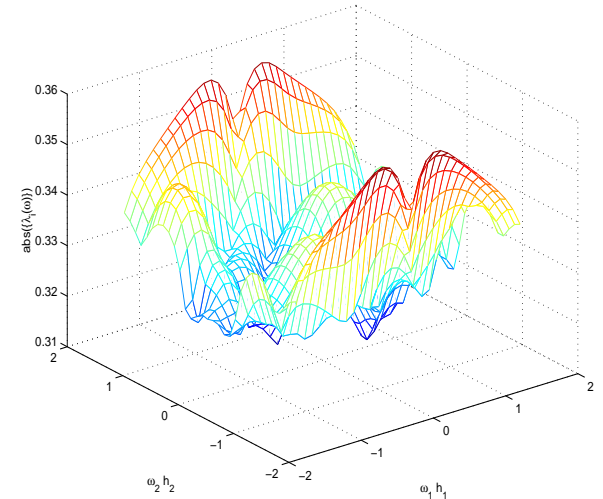

Baum-DG,

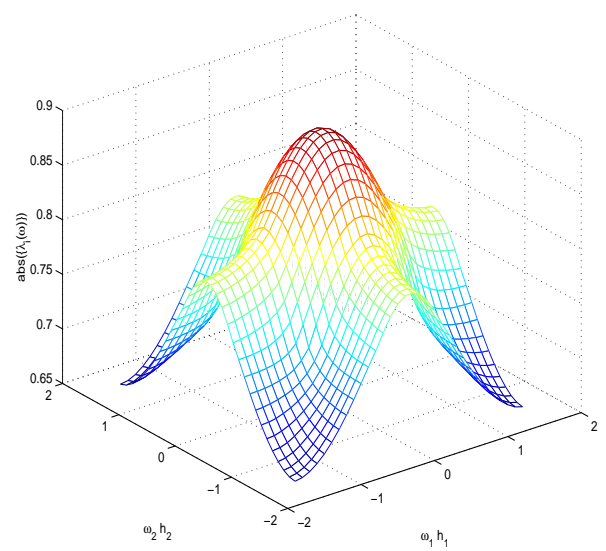

Symm-DG, $\rho\left(\widehat{M_{h}^{C G C}}(\omega) \widehat{M_{J O R}^{R E L}}(\omega)\right)$,

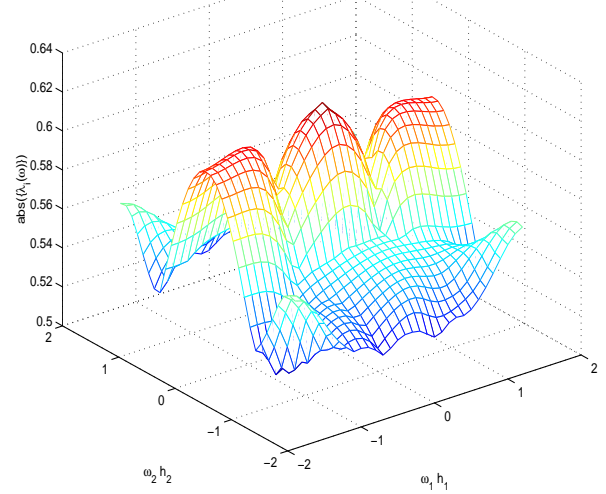

Symm-DG, $\rho\left(\widehat{M_{h}^{C G C}}(\omega) \widehat{M_{D G S}^{R E L}}(\omega)\right)$,

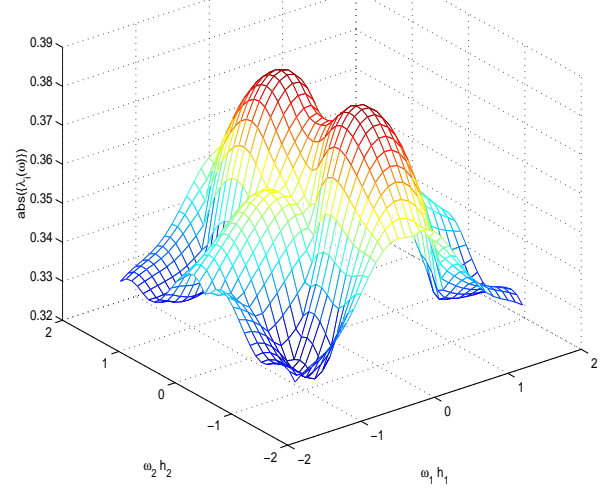

Symm-DG,

$$
\rho\left(\widehat{M_{D G S_{U}}^{R E L}}(\omega) \widehat{M_{h}^{C G C}}(\omega) \widehat{M_{D G S_{L}}^{R E L}}(\omega)\right), \quad \rho\left(\widehat{M_{D G S_{U}}^{R E L}}(\omega) \widehat{M_{h}^{C G C}}(\omega) \widehat{M_{D G S_{L}}^{R E L}}(\omega)\right),
$$

Figure 5: Spectral radii $\left(\max _{j}\left(\left|\lambda_{j}(\omega)\right|\right), j \in\{1,2, . ., 12\}\right)$ as function of $\omega \in \mathbb{T}_{h}^{2}$ for the symmetric $(\sigma=-1)$ and Baumann's DG-method $(\sigma=1)$ for damping parameters as in Table 3. 


\begin{tabular}{|c|c|c|}
\hline$\alpha_{\text {opt }}$ & Baum-DG & symm-DG \\
\hline$M_{h}^{C G C} M_{J O R}^{R E L}$ & 0.95 & 1.03 \\
\hline$M_{h}^{C G C} M_{D G S}^{R E L}$ & 1.22 & 1.44 \\
\hline
\end{tabular}

Table 3: Optimal damping parameters, $\alpha_{\text {opt }}$, for the two-level operators $\rho\left(M_{h}^{C G C} M_{h}^{R E L}\right)=$ $\rho\left(\bar{M}_{h}^{R E L} \bar{M}_{h}^{C G C}\right)$.

\begin{tabular}{|c|c|c|}
\hline$\rho\left(M_{h}^{C G C} M_{h}^{R E L}\right)$ & Baum-DG & symm-DG \\
\hline$M_{h}^{C G C} M_{J O R}^{R E L}$ & 0.74 & 0.89 \\
\hline$M_{h}^{C G C} M_{D G S}^{R E L}$ & 0.44 & 0.62 \\
\hline$M_{D G S_{U}}^{R E L} M_{h}^{C G C} M_{D G S_{L}}^{R E L}$ & 0.36 & 0.38 \\
\hline
\end{tabular}

Table 4: Spectral radii $\rho\left(M_{h}^{C G C} M_{h}^{R E L}\right)=\rho\left(\bar{M}_{h}^{R E L} \bar{M}_{h}^{C G C}\right)$ for optimal damping parameters as in Table 3.

\begin{tabular}{|c|c|c|c|}
\hline & $\left(\bar{M}_{J O R}^{R E L} \bar{M}_{h}^{C G C}\right)^{k}$ & $\left(\bar{M}_{D G S_{L}}^{R E L} \bar{M}_{h}^{C G C}\right)^{k}$ & $\left(\bar{M}_{D G S_{L}}^{R E L} \bar{M}_{h}^{C G C} \bar{M}_{D G S_{U}}^{R E L}\right)^{k}$ \\
\hline Baum-DG, $k=1$ & 3.15 & 3.48 & 2.37 \\
\hline Baum-DG, $k=3$ & 1.02 & 0.72 & 0.34 \\
\hline Baum-DG, $k=4$ & 0.68 & 0.32 & 0.13 \\
\hline \hline Symm-DG, $k=1$ & 7.46 & 5.82 & 0.09 \\
\hline Symm-DG, $k=3$ & 2.65 & 1.74 & 0.36 \\
\hline Symm-DG, $k=4$ & 2.24 & 1.01 & 0.31 \\
\hline
\end{tabular}

Table 5: The spectral norm $\left\|\left(\bar{M}^{R E L} \bar{M}_{h}^{C G C}\right)^{k}\right\|_{2}$ for the amplification operator of the residue with optimal damping, $k=1,3,4$.

with on the Dirichlet boundary $\partial \Omega$

$$
u(x, y)=\frac{2-\left(e^{x / \epsilon}+e^{y / \epsilon}\right)}{e^{1 / \epsilon}-1}+x+y .
$$

To obtain the discrete system we use the reduced polynomial basis for $S_{h}$ as explained in section 4.2. I.e. we use for each cell $\Omega_{e}$ a local basis consisting of $\phi_{e, i}(\xi) \phi_{e, j}(\eta)$ as in 2.7 , where $(i, j) \in\{(m . n),(m+2, n),(m, n+2) \mid m, n=0,1\}$. We use a regular mesh with size $h \times h=4^{-N}$ and we start with an initial function $u_{h}^{0}(x, y)=u_{P R E}^{0}$ on the finer grid. We apply $\nu_{1}$ pre-relaxation sweeps

$$
u_{h, \mathrm{PRE}}^{i+1}=u_{h, \mathrm{PRE}}^{i}+B_{h}\left(f_{h}-L_{h} u_{h, \mathrm{PRE}}^{i}\right),
$$

where $B_{h}$ is the approximate inverse of $L_{h}$ as given in Table 1 . Then, we update the solution by a coarse grid correction step, solving for $e_{H}$ on the coarser grid with size $H \times H=4^{1-N}$,

$$
u_{h, \mathrm{POST}}^{0}=u_{h, \mathrm{PRE}}^{\nu_{1}}+P_{h H} L_{H}^{-1} \bar{R}_{H h}\left(f_{h}-L_{h} u_{h, \mathrm{PRE}}^{\nu_{1}}\right) .
$$




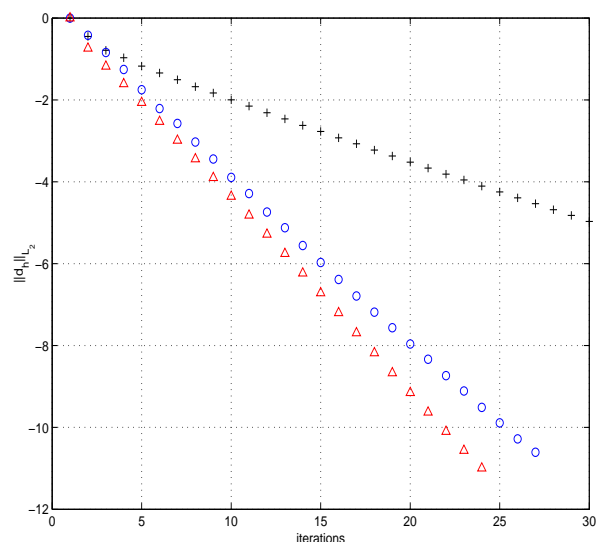

Baumann-DG

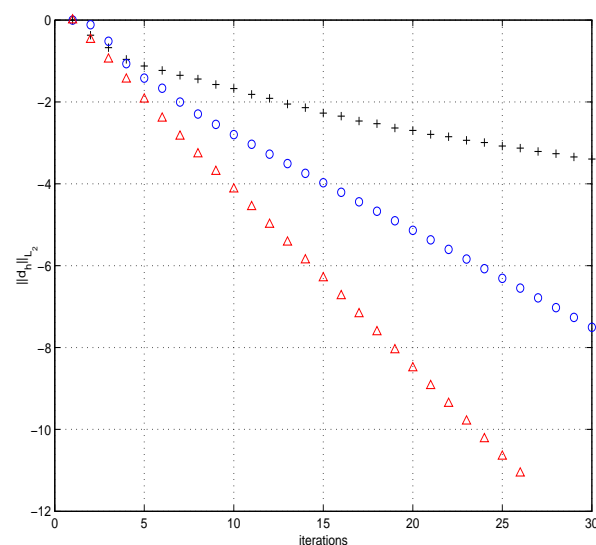

Symmetric-DG

$$
\text { +: JOR; ०: DGS; } \triangle \text { : SGS. }
$$

Figure 6: $\log \left(\left\|d_{h}\right\|_{L_{2}}\right)$ as function of iterations for the two-level iteration operator on the error

and, eventually, we apply $\nu_{2}$ post-relaxations sweeps

$$
u_{h, \mathrm{POST}}^{i+1}=u_{h, \mathrm{POST}}^{i}+B_{h}\left(f_{h}-L_{h} u_{h, \mathrm{POST}}^{i}\right),
$$

to compute $u_{h}^{i+1}=u_{h \text {,POST }}^{\nu_{2}}$. The correction on the coarser grid at its turn, is solved by multigrid until the residue of the correction (in the $L_{2}$ norm) is less than an order of $O\left(10^{-6}\right)$. To show convergence we measure the residue in the $L_{2}$ norm $^{3}$

$$
\left\|d_{h}\right\|_{L_{2}}=\left\|f_{h}-L_{h} u_{h}\right\|_{L_{2}}=\left(\sum_{e} \int_{\Omega_{e}}\left|\sum_{i=0}^{12} c_{e, i} \phi_{e, i}(x, y)\right|^{2} d x\right)^{1 / 2} .
$$

The observed convergence of the two-level solution method applied to Baumann's and the symmetric DG-method are shown in Figure 6. We observe that both methods show convergence, however Baumann's DG-method converges faster. From the slope we estimate the convergence rates for the different two-level algorithms. Table 6 shows the results and we see that the observed rates in the numerical experiments coincide well with the spectral radii obtained by Fourier analysis as shown in Table 4 .

\section{Conclusion}

In this paper we analyze the convergence behavior of the two-level algorithm applied to the two-dimensional Poisson equation, discretized by two discontinuous Galerkin (DG) methods: the Baumann-Oden and the symmetric DG-method, each with a polynomial basis of piecewise cubics in each of the two coordinate directions. We studied the convergence behavior of different block relaxation methods: damped block Jacobi (JOR), damped block Gauss-Seidel (DGS) and symmetric damped block Gauss Seidel (SDGS), where the blocks

\footnotetext{
${ }^{3}$ According to (3.5) we would follow the Fourier analysis more precisely if we would measure the residue in the vector two-norm, however both norms are equivalent.
} 


\begin{tabular}{|c|c|c|}
\hline$\rho\left(M_{h}^{C G C} M_{h}^{R E L}\right)$ & Baum-DG & symm-DG \\
\hline$M_{h}^{C G C} M_{J O R}^{R E L}$ & 0.7 & 0.9 \\
\hline$M_{h}^{C G C} M_{D G S}^{R E L}$ & 0.4 & 0.6 \\
\hline$M_{D G S_{U}}^{R E L} M_{h}^{C G C} M_{D G S_{L}}^{R E L}$ & 0.3 & 0.4 \\
\hline
\end{tabular}

Table 6: Numerically obtained convergence rates observed for the different two-level blockrelaxation methods with optimal damping parameters as in Table 3.

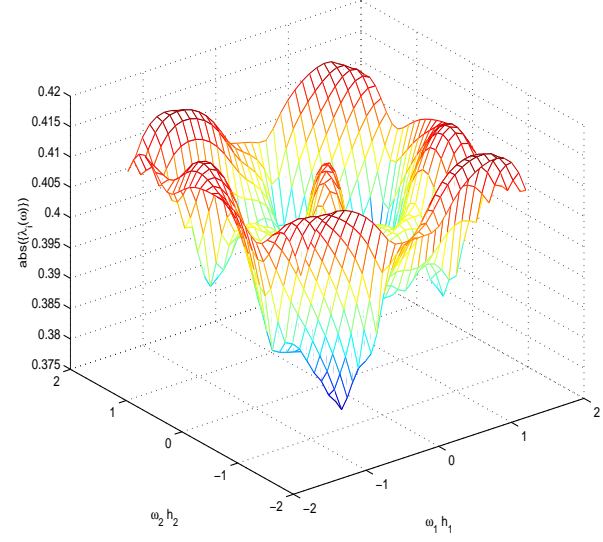

Baumann-DG

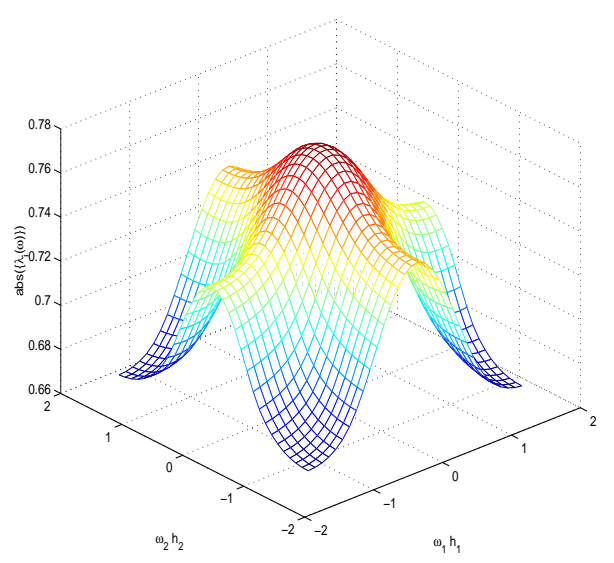

Symmetric-DG

Figure 7: $\rho\left(\widehat{M_{D G S_{U}}^{R E L}}(\omega) \widehat{M_{h}^{C G C}}(\omega) \widehat{M_{D G S_{L}}^{R E L}}(\omega)\right)$

are chosen, based either on cell-wise or on point-wise ordering. We show that point-wise block relaxation has better smoothing properties than the classical cell-wise block relaxation methods. Moreover, point-wise block relaxation for the symmetric DG-method is stable, whereas the classical cell-wise relaxation methods are not.

The smoothing behavior is further improved by reduction of the polynomial basis, i.e. removing tensor-basis functions that represent cross derivatives at the cell corners, but do not contribute to the order of accuracy. Reduction of the basis not only improves the convergence behavior of the relaxation methods, it also makes the DG-method much more efficient than when it is based on a tensor-product basis.

For the two-level algorithm we computed optimal damping parameters for the relaxation methods and spectral radii of the corresponding iteration operators. With a spectral radius between 0.6 and 0.4 for DGS and SDGS smoothers, the two-level algorithms show good convergence. An analysis of the spectral norm on the residual shows that residual reduction is guaranteed within 4 iteration steps. 


\begin{tabular}{|l|l|l|}
\hline$\rho\left(M_{D G S_{U}}^{R E L} M_{h}^{C G C} M_{D G S_{L}}^{R E L}\right)$ & 0.42 & 0.77 \\
\hline
\end{tabular}

Table 7: Spectral radii for optimal damping parameters as in Table 3.

\begin{tabular}{|l|l|l|}
\hline$\rho\left(M_{D G S_{U}}^{R E L} M_{h}^{C G C} M_{D G S_{L}}^{R E L}\right)$ & 0.4 & 0.7 \\
\hline
\end{tabular}

Table 8: Numerical obtained convergence rates for the symmetric block relaxation methods with symmetric damping and optimal damping parameters from Table 3.

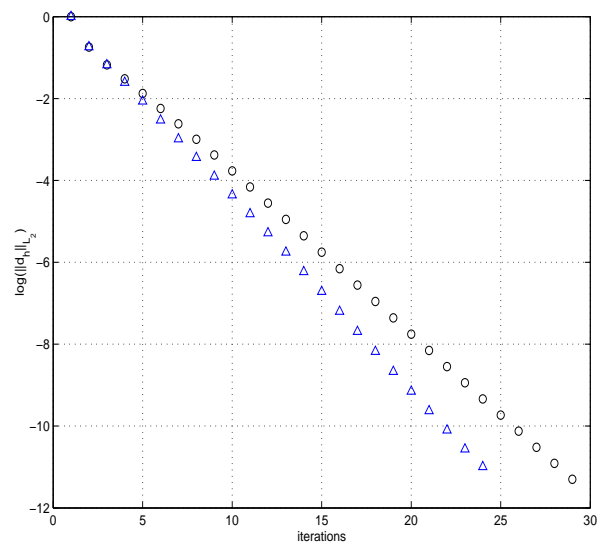

Baumann-DG

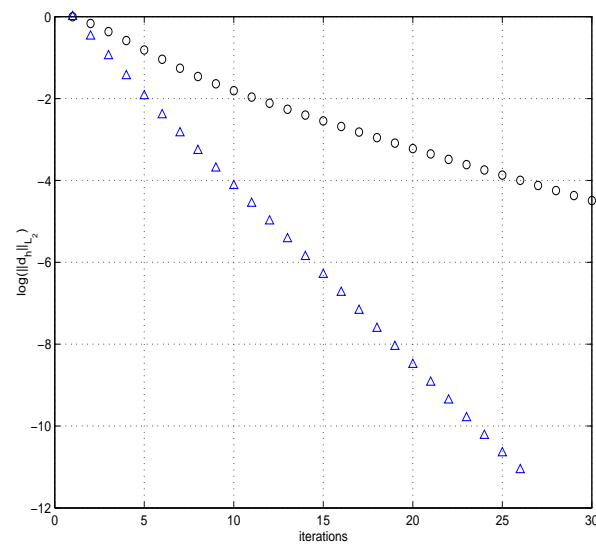

Symmetric-DG ०: SSGS; $\triangle$ : SGS.

Figure 8: $\log \left(\left\|d_{h}\right\|_{L_{2}}\right)$ as function of iterations for the two-level iteration operator on the error

\section{REFERENCES}

1. M. Abramowitz and I.A. Stegun. Handbook of mathematical functions. Dover Publ. Inc., 1964.

2. D. N. Arnold. An interior penalty finite element method with discontinuous elements. SIAM J. Numer. Anal., 19:742-760, 1982.

3. D.N. Arnold, F. Brezzi, B. Cockburn, and L.D. Marini. Unified analysis of discontinuous Galerkin methods for elliptic problems. SIAM J Numer. Anal., 39:1749-1779, 2002. to appear.

4. P. Bastian and V. Reichenberger. Multigrid for higher order discontinuous Galerkin finite elements applied to groundwater flow. Technical Report 2000-37, SFB 359, 2000.

5. J.H. Bramble. Multigrid Methods. Pitman Research Notes in Mathematics Series 294. Longman Scientific \& Technical, Harlow, 1993.

6. L. Delves and C. Hall. An implicit matching principle for global element calculations. $J$. Inst. Math. Appl., 23:223-234, 1979.

7. J. Gopalakrishnan and G. Kanschat. A multilevel discontinuous Galerkin method. Preprint 1735, IMA, dec 2000. submitted to Numerische Mathematik. 
8. P. W. Hemker. Fourier analysis of gridfunctions, prolongations and restrictions. Technical Report NW 93, Mathematical Centre, Amsterdam, 1980.

9. P.W. Hemker, W. Hoffmann, and M.H. van Raalte. Fourier two-level analysis of a multigrid approach for discontinuous galerkin discretisation with linear elements. Technical Report MAS-R0217, CWI, Amsterdam, June 2002. submitted to NLAA.

10. P.W. Hemker, W. Hoffmann, and M.H. van Raalte. Two-level fourier analysis of a multigrid approach for discontinuous galerkin discretisation. Technical Report MAS-R0206, CWI, Amsterdam, April 2002. submitted to SIAM SISC.

11. J. Hendry and L. Delves. The global element method applied to a harmonic mixed boundary value problem. J. Comp. Phys., 33:33-44, 1978.

12. J. Nitsche. Über ein Variationsprinzip zur Lösung von Dirichlet Problemen bei Verwendung von Teilraumen die keinen Randbedingungen unterworfen sind. Abh. Math. Sem. Univ. Hamburg, 36:9 15, 1971.

13. J.T. Oden, I. Babuška, and C.E. Baumann. A discontinuous $h p$ finite element method for diffusion problems. J. Comp. Phys., 146:491-519, 1998.

14. M. Wheeler. An elliptic collocation-finite element method with interior penalties. SIAM J. Numer. Anal., 15:152-161, 1978. 